\title{
The Evolution of Optical OFDM
}

\author{
Xiaoyu Zhang, Member, IEEE, Zunaira Babar, Senior Member, IEEE, Periklis Petropoulos, \\ Harald Haas, Fellow, IEEE, and Lajos Hanzo, Fellow, IEEE
}

\begin{abstract}
Optical Orthogonal Frequency-Division Multiplexing (O-OFDM) is eminently suitable for mitigating the multi-path and chromatic dispersion in both Visible Light Communications (VLC) and Optical Fiber Communications. We commence our discourse by surveying the conception and historic evolution of O-OFDM designed for both VLC and optical fiber, culminating in the birth of its most flexible design alternative, namely Layered Asymmetrically Clipped Optical OFDM (LACO-OFDM). We demonstrate that it is eminently suitable for intensity-modulation and direct-detection aided optical communication systems and characterize its design flexibility. It is also shown that given its flexibility, it subsumes a wide range of optical OFDM schemes conceived over the past two decades or so. The LACO-OFDM transmitter and receiver designs strike a compelling compromise between the features of the popular Asymmetrically Clipped Optical OFDM (ACO-OFDM) and Direct Current Offset OFDM (DCO-OFDM). The pivotal role of forward error correction (FEC) designs is also surveyed with the objective of striking a coding gain versus complexity trade-off. We conclude by highlighting a suite of promising techniques capable of further improving the system performance, but require further research. The take-away message of the paper crystallized in the associated design guidelines.
\end{abstract}

Index Terms-LACO-OFDM, transceiver design, signal characterization, performance analysis, forward error correction.

\section{INTRODUCTION}

\section{A. OFDM for Intensity-Modulated Direct-Detection Communications}

A $\mathrm{S}$ an attractive modulation technique originally conceived in 1966 [1], Orthogonal Frequency Division Multiplexing (OFDM) has been widely researched and developed for Radio Frequency (RF) applications [2]-[7]. As an off-shoot of RFOFDM, Optical OFDM (O-OFDM) has been proposed for the Visible Light Communications (VLC) downlink, as a benefit of its ability to mitigate the multipath-induced inter-symbol interference [8]. However, O-OFDM has had a shorter history

L. Hanzo would like to acknowledge the financial support of the Engineering and Physical Sciences Research Council projects EP/P034284/1 and EP/P003990/1 (COALESCE) as well as of the European Research Council's Advanced Fellow Grant QuantCom (Grant No. 789028).

$\mathrm{X}$. Zhang is with both the School of Electronics and Computer Science and the Optoelectronics Research Centre, University of Southampton, Southampton, SO17 1BJ, U.K. (e-mail: xy.zhang@ soton.ac.uk).

Z. Babar is with Viavi Solutions, Stevenage, SG1 2AN, U.K, and also with the School of Electronics and Computer Science, University of Southampton, Southampton, SO17 1BJ, U.K. (e-mail: zunaira.babar@gmail.com).

P. Petropoulos is with the Optoelectronics Research Centre, University of Southampton, Southampton, SO17 1BJ, U.K. (e-mail: pp@orc.soton.ac.uk).

H. Haas is with the LiFi Research and Development Centre, School of Engineering, Institute for Digital Communications, The University of Edinburgh, Edinburgh, EH9 3JL, U.K. (e-mail: h.haas@ed.ac.uk).

L. Hanzo is with the School of Electronics and Computer Science, University of Southampton, Southampton, SO17 1BJ, U.K. (e-mail: 1h@ecs.soton.ac.uk).
TABLE I

COMPARISON OF TYPICAL SYSTEM CHARACTERISTICS IN RF-OFDM AND O-OFDM

\begin{tabular}{lrr}
\hline \multicolumn{1}{c}{ Topic } & RF-OFDM & O-OFDM \\
\hline TX Signal & bipolar and complex-valued & unipolar \\
Media & RF electromagnetic waves & lightwave \\
Detection & (majorly) coherent & direct detection \\
\hline
\end{tabular}

than RF-OFDM [8]-[11] and has only had a rapid evolution in the recent two decades.

In contrast to classic RF-OFDM, the low-cost method of Intensity-Modulation paired with Direct-Detection (IM/DD) requires the signal to be positive. Hence, a substantial amount of research efforts have been devoted to the improvement of O-OFDM schemes, with the objective of striking a tradeoff between the Power- and Bandwidth Efficiency (BE and $\mathrm{PE})$. Some of the most important distinctions between RFOFDM and O-OFDM are summarized in Tab. I. In [12], Kahn and Barry characterized the pysical behavior of infrared channels using IM/DD. In IM/DD systems, the signal to be transmitted modulates the intensity of the emitted lightwave. At the receiver, a Photo Detector (PD) is used for converting the optical signal into electronic current for representing the received signal. The resultant IM/DD systems have been widely employed both in optical wireless [13], [14] and in fiber-optic communications [15].

However, the optical signals must be real- and positivevalued, whereas conventional baseband OFDM signals relying on Fast Fourier Transform (FFT)-based modulation are typically bipolar- and complex-valued. Upon satisfying the constraint of Hermitian symmetry in the Frequency Domain (FD), the Time Domain (TD) counterpart of the OFDM signal after Inverse FFT (IFFT) is guaranteed to be real-valued, but we still have to eliminate the negative samples. To circumvent this problem, DC-Biased Optical OFDM (DCO-OFDM) [16], [17] and Asymmetrically Clipped OFDM (ACO-OFDM) [18], [19] have been proposed.

\section{B. DCO-OFDM vs ACO-OFDM}

Carruthers and Kahn [16] have proposed to positively bias the bipolar real-valued signal generated after satisfying the Hermitian symmetry with the aid of a DC component, so that the signal became positive, hence the terminology of DCO-OFDM. Since the magnitude of the signal is randomly distributed, some small residual negative samples may persist after adding the DC offset. Therefore, the DC offset to be used must be carefully decided, so that the damage to the integrity of the information conveyed remains negligible after clipping 
the negative residual samples. As a common practice [19], the DC offset of DCO-OFDM is typically in excess of $10 \mathrm{~dB}$ higher than the information-bearing bipolar signal.

In order to avoid the above-mentioned substantial powerloss imposed by the DC-bias, Armstrong and Lowery [18] proposed the ACO-OFDM concept, where the bipolar modulated signal is directly clipped at zero after IFFT. Explicitly, this is achieved by arranging for the FD OFDM signals so that only their odd-indexed subcarriers are non-zero, hence creating an opportunity for all the distortions generated by clipping the negative samples to fall solely on the even-indexed ones. This specific measure allows ACO-OFDM to retain information integrity, despite the drastic clipping process.

Moreover, we would also like to mention that similar characteristic features are exhibited by the Pulse-AmplitudeModulated Discrete MultiTone (PAM-DMT) scheme, where the imaginary part of all subcarriers is actively modulated, while the real part is left blank [20]. Other diverse schemes have also been advocated for avoiding the DC energy wastage, such as Flip-OFDM/U-OFDM [21], [22], which transmit the positive and inverted negative parts of the real-valued symbols separately in the TD.

\section{Hybrid O-OFDM Schemes}

In terms of practical implementations conceived for striking a trade-off between the bandwidth and power efficiency, a rich variety of designs can be found in the literature [23][28]. They are commonly termed as hybrid schemes, since they tend to have multiple basic unipolar OFDM schemes (e.g. ACO-OFDM, DCO-OFDM, PAM-DMT, etc.) in their structures. The Asymmetrically Clipped DC Biased Optical OFDM (ADO-OFDM) scheme was proposed by Dissanayake et al. [24], which utilizes both ACO- and DCO- OFDM by multiplexing the two schemes in the FD. Relying on a similar multiplexing philosophy, Ranjha and Kavehrad [25] proposed the Hybrid Asymmetrically Clipped Optical OFDM (HACOOFDM) scheme, combining ACO-OFDM and PAM-DMT. In [27], a more advanced hybrid system combining ACOOFDM and PAM-DMT was proposed, under the terminology of Asymmetrical Hybrid Optical OFDM (AHO-OFDM). In contrast to HACO-OFDM, one of the two constituent signals in AHO-OFDM must be inverted, yielding a bipolar signal, which requires additional DC bias for eliminating the negative samples. Moreover, Asymmetrically and Symmetrically Clipped Optical OFDM (ASCO-OFDM) was proposed by Wu and Bar-Ness [26], which relies on an idea equivalent to multiplexing ACO-OFDM and Flip-OFDM in the FD. In [23], a pair of hybrid schemes, namely the Absolute Value Optical OFDM (AVO-OFDM) and Asymmetrically Clipped Absolute Value Optical OFDM (AAO-OFDM) were proposed. The AVO-OFDM scheme transmits the absolute value of the bipolar Hermitian-symmetry-constrained signal and uses additional resources to transmit their sign information. On the other hand, AAO-OFDM amalgamates AVO-OFDM and ACO-OFDM for attaining an improved performance.

Before we proceed, Tab. II summaries all the O-OFDM schemes ${ }^{1}$ that have been mentioned up to this stage. The last column of Tab II shows the corresponding typical BE, when compared to its RF counterpart having the same bandwidth. Observe from Tab. II that

- Only DCO-OFDM as well as the hybrid schemes relying on it, and AHO-OFDM can achieve the BE of $\frac{1}{2}$, but unfortunately all of them require DC offset;

- The hybrid schemes have fixed BE values, which is dependent on the specific choice of its constituent schemes.

A further design option is constituted by the recently proposed Layered ACO-OFDM (LACO-OFDM) [29], which populates the idle subcarriers of ACO-OFDM by creating additional layers and mapping extra sets of symbols to the originally un-populated positions of the ACO-OFDM scheme.

\section{Evolution of LACO-OFDM}

The concept of exploiting a layered structure has been widely adopted in communications systems [30]-[32] and it is also natural to introduce this idea for improving the IM/DD physical layer.

The original concept of LACO-OFDM was first published in a journal by Wang et al. [29] in May 2015, but Tsonev and Haas presented a previous conference on layered modulation at the 50th IEEE International Conference on Communications in 2014 [33], and followed it up by a journal publication [34] in Sep. 2015, where U-OFDM rather than ACO-OFDM was employed at each layer. Both author teams quantified the $\mathrm{BE}$ improvement attained, which eventually converges to that of the DCO-OFDM scheme, as the number of layers is increased. The Bit Error Ratio (BER) performance was also quantified for transmission over AWGN channels under limited electric and optical powers. During the same era, three other schemes utilizing a similar philosophy were also reported in the literature.

- Elgala and Little [35] quantified both the throughput and Peak-to-Average Power Ratio (PAPR) of their scheme, which was termed as Spectral- and Energy-Efficient OFDM (SEE-OFDM).

- As a further development, in [36] the idea of superimposing multiple streams was proposed by Islim et al., which was then applied to ACO-OFDM. The authors studied the spectral efficiency of the superimposed signal, where the length of the OFDM Cyclic Prefix (CP) was also considered. It was demonstrated that the SE approached that of the DCO-OFDM. Therefore, they referred to this scheme as enhanced ACO-OFDM (eACO-OFDM). The BER performance of eACO-OFDM was also studied and it was contrasted to that of DCO-OFDM and ePAM-DMT [37].

- Lowery [38] metaphorized the symbol-to-subcarrier loading process as the arrangement of notes into chords of different octaves in music. On a particular octave, only specific harmonics could be loaded with notes, which resembled the ACO-OFDM philosophy. The SE improvement attained was calculated and compared to

${ }^{1}$ PAM-DMT is classified here as an O-OFDM for the sake of comparison. 
TABLE II

LIST OF BASIC AND HYBRID O-OFDM SCHEMES INTRODUCED

\begin{tabular}{cccclc}
\hline Nature & Time & Ref. & Name & \multicolumn{1}{c}{ Design } & BE \\
\hline \multirow{6}{*}{ Basic } & Apr. 1996 & {$[16]$} & DCO-OFDM & DC offset & $1 / 2$ \\
& Mar. 2006 & {$[18]$} & ACO-OFDM & Clipping at zero, idle subcarrier for distortion & $1 / 4$ \\
& Dec. 2009 & {$[20]$} & PAM-DMT & Clipping at zero, only imaginary part in FD & $1 / 4$ \\
& May. 2012 & {$[22]$} & U-OFDM & Transmit positive and negative parts separately & $1 / 4$ \\
& Dec. 2012 & {$[21]$} & Flip-OFDM & Transmit positive and negative parts separately & $1 / 4$ \\
& Sep. 2017 & {$[23]$} & AVO-OFDM & Transmit magnitude and sign parts separately & $<1 / 2$ \\
\hline \multirow{4}{*}{ Hybrid } & Dec. 2011 & {$[24]$} & ADO-OFDM & ACO-OFDM + DCO-OFDM & $1 / 2$ \\
& Apr. 2014 & {$[25]$} & HACO-OFDM & ACO-OFDM + PAM-DMT & $3 / 8$ \\
& Jan. 2015 & {$[26]$} & ASCO-OFDM & ACO-OFDM + Flip-OFDM & $3 / 8$ \\
& May 2015 & {$[27]$} & AHO-OFDM & ACO-OFDM + PAM-DMT + DC & $1 / 2$ \\
& Sep. 2017 & {$[23]$} & AAO-OFDM & ACO-OFDM + AVO-OFDM & $<1 / 2$ \\
\hline
\end{tabular}

that of ACO-OFDM, leading to the terminology of Enhanced ACO-OFDM (EACO-OFDM). The performance of EACO-OFDM was characterized by the Error Vector Magnitude (EVM) metric through simulations and it was contrasted to both PAM-DMT and ADO-OFDM.

In the past half decade, LACO-OFDM has attracted much attention and many further developments have been published, which are summarized in Tab. III, where the dates of (print) publications and their main contributions are listed.

In this paper, we focus our attention on the fundamental design, characterization and error correction of LACO-OFDM and its relationship with the parental ACO-OFDM, while other notable breakthroughs from Tab. III will also be covered in Section VI.

\section{E. Novel Contributions and Paper Structure}

Several survey papers have been published comparing the performance of LACO-OFDM to other O-OFDM schemes [44], [51], [73], [74]. Their contributions are summarized and boldly contrasted to our work in Tab. IV. Explicitly, the mathematical BER and PAPR performance analyses of various O-OFDM schemes was carried out by Sun et al. [44], while a survey authored by Lowery [74] focused on the associated transceiver signal processing and spectral analysis. However, the family of error correction techniques designed for $\mathrm{O}$ OFDM schemes - which is a vitally important component of any practical implementation - has hardly been touched upon in the related survey papers. Against this background, this paper presents an overview of LACO-OFDM, with an emphasis on its salient characteristics distinguishing it from other O-OFDM schemes, as well as on its error correction techniques. The outline of this treatise is given below:

Section II provides the required background information and outlines the challenges of optical communication schemes. Section III discusses the transmitter design and transmit signal of LACO-OFDM, while Section IV presents the receiver design and its performance analysis. Section $\mathrm{V}$ focuses on the design options of error correction methods conceived

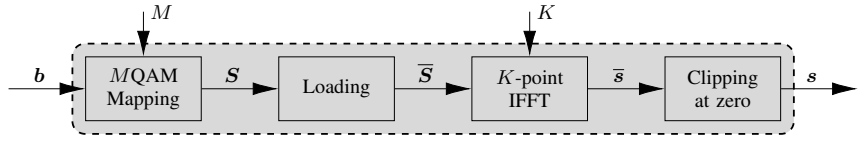

Fig. 1. Transmitter block of ACO-OFDM (ACO TX).

for LACO-OFDM. In Section VI, additional techniques of improving the performance of LACO-OFDM are considered. Finally, in Section VII, our conclusions and general design guidelines are provided for LACO-OFDM.

\section{BACKGROUND AND CHALLENGES}

In this section, we introduce the schematic of ACO- and DCO-OFDM as background information, and discuss their bandwidth- vs. power-efficiency.

\section{A. Asymmetrically Clipped Optical OFDM}

1) ACO-OFDM Transmitter: Fig. 1 shows the basic schematic of the ACO-OFDM transmitter, where $M$-ary Quadrature Amplitude Modulation (MQAM) and $K$-point IFFT are adopted. Given a total of $K$ subcarriers, only a quarter of them $(K / 4)$ can be used for conveying complexvalued message symbols for transporting the bit stream $\boldsymbol{b}$. Another set of $K / 4$ subcarriers convey their conjugated counterparts loaded, while the remaining $K / 2$ subcarriers carry null symbols. More specifically, the above-mentioned strategy for loading the stream $\boldsymbol{S}$ of Fig. 1 onto $\overline{\boldsymbol{S}}$ can be expressed by:

$$
\begin{aligned}
& \bar{S}[k]= \begin{cases}S[u], & k=2 u+1, \\
S^{*}[u], & k=K-(2 u+1), \\
0, & \text { otherwise, }\end{cases} \\
& 0 \leq u \leq \frac{K}{4} \quad \text { and } \quad 0 \leq k \leq K-1,
\end{aligned}
$$

where ${ }^{*}$ represents the conjugation operation for complex numbers.

As a result of the Hermitian symmetry in $\bar{S}$, its TD representation of $\bar{s}$ produced by the IFFT operation of Fig. 1 is real-valued and anti-symmetric:

$$
-\bar{s}[n]=\bar{s}\left[\frac{K}{2}+n\right], \quad 0 \leq k<\frac{K}{2} .
$$


TABLE III

LACO-OFDM RESEARCH TIMELINE

\begin{tabular}{|c|c|c|}
\hline Mar. 2006 & [39] & Proposal and experimental demonstration of OFDM based VLC \\
\hline Nov. 2007 & [40] & Indoor O-OFDM channel modeling and performance analysis \\
\hline Sep. 2014 & [35] & Independent proposition of SEE-OFDM \\
\hline May 2015 & [29] & The proposition of LACO-OFDM \\
\hline Dec. 2015 & [36] & Independent proposition of eACO-OFDM \\
\hline Feb. 2016 & [41] & Improved clipping distortion cancellation at $\mathrm{RX}$ \\
\hline Mar. 2016 & [38] & Independent proposition of EACO-OFDM \\
\hline \multirow[t]{3}{*}{ Jun. 2017} & [42] & RX with diversity combining and SIC \\
\hline & [43] & $\mathrm{RX}$ with diversity combining and clipping distortion recovery \\
\hline & [44] & Performance analysis and comparison \\
\hline Aug. 2017 & [45] & Performance analysis and PAPR reduction \\
\hline Sep. 2017 & [46] & Channel coding and experiment \\
\hline Dec. 2017 & [47] & Capacity analysis and adaptive modulation \\
\hline Jan. 2018 & [48] & SCFDM and reduced PAPR \\
\hline Apr. 2018 & [49] & Capacity analysis \\
\hline Jun. 2018 & [50] & Improved RX with SIC \\
\hline Oct. 2018 & [51] & Performance analysis \\
\hline \multirow[t]{3}{*}{ Jan. 2019} & [52] & Improved RX on clipping noise mitigation \\
\hline & [53] & Capacity analysis, channel coding \\
\hline & [54] & SCFDM and dimming control \\
\hline Feb. 2019 & [55] & Dimming control \\
\hline \multirow[t]{2}{*}{ Mar. 2019} & [56] & Non-Orthogonal Multiple Access (NOMA) in LACO-OFDM and network scheduling \\
\hline & [57] & NOMA and hierarchical pre-distorted TX design \\
\hline Apr. 2019 & [58] & Channel coding \\
\hline May 2019 & [59] & Improved spectral efficiency with additional hybrid layer \\
\hline Jun. 2019 & [60] & TX side improvement with precoding \\
\hline Aug. 2019 & [61] & Multi-input TX design \\
\hline Oct. 2019 & [62] & Adaptive modulation based on [57] \\
\hline Jan. 2020 & [63] & Triple-layer hybrid O-OFDM with improved BER performance, spectral and energy efficiency \\
\hline Apr. 2020 & [64] & Joint PAPR and complexity reduction design \\
\hline Jul. 2020 & [65] & Further analysis and experimental demonstration of [63] \\
\hline Aug. 2020 & [66] & Transmission considering LED nonlinearity \\
\hline \multirow[t]{4}{*}{ Sep. 2020} & [67] & $\mathrm{RX}$ design with no iterative detection requirement \\
\hline & [68] & Capacity analysis and optimization \\
\hline & [69] & Channel coding and optical fiber experiment \\
\hline & [70] & Error rate performance improvement with analysis on residual clipping noise \\
\hline Nov. 2020 & [71] & Design with adaptive modulation and reduced detection latency \\
\hline Later 2021 & [72] & Index Modulation \\
\hline
\end{tabular}


TABLE IV

A COMPARISON OF SURVEYS AND TUTORIALS ON THE LACO-OFDM SCHEME.

\begin{tabular}{|c|c|c|c|c|c|}
\hline Topic in LACO-OFDM & [44] & [51] & [73] & [74] & Our Work \\
\hline \hline Transceiver Schematic & $\checkmark$ & & & $\checkmark$ & $\checkmark$ \\
\hline Detailed Signal Flow & & $\checkmark$ & & $\checkmark$ & $\checkmark$ \\
\hline BER Performance & $\checkmark$ & & & & $\checkmark$ \\
\hline PAPR Performance & $\checkmark$ & & & & $\checkmark$ \\
\hline $\begin{array}{l}\text { Performance Comparison } \\
\text { to other O-OFDM }\end{array}$ & $\checkmark$ & $\checkmark$ & $\checkmark$ & $\checkmark$ & $\checkmark$ \\
\hline Error Correction Methods & & & & & $\checkmark$ \\
\hline
\end{tabular}

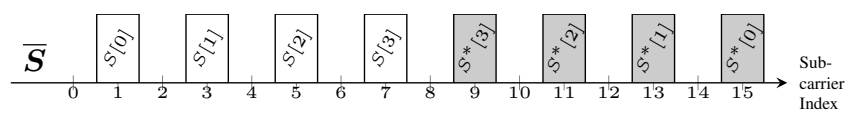

(a) Loaded FD Signal

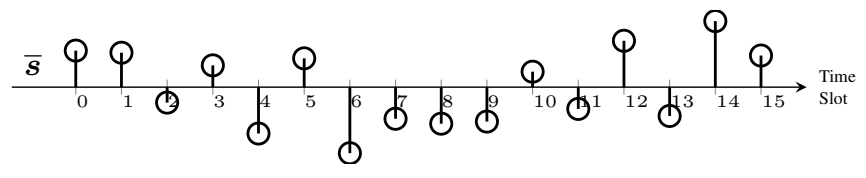

(b) Loaded TD Signal

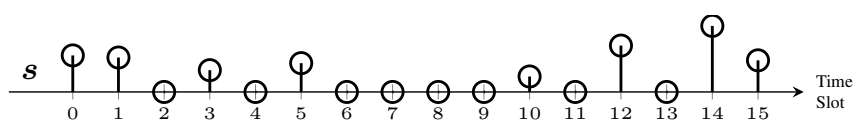

(c) Clipped TD Signal

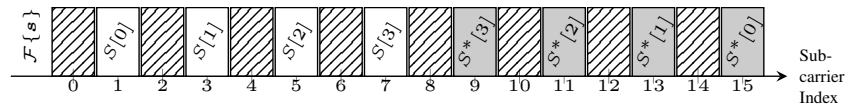

(d) Clipped FD Signal, where the hatched boxes represent clipping distortion

Fig. 2. Example of ACO-OFDM TX process signal flow.

Hence, negative time samples in $\bar{s}$ can be dropped without any information being lost. The resultant real-valued non-negative (i.e. unipolar) electric signal $s$ can then be transmitted by optical intensity modulation.

2) ACO-OFDM Signals: In Fig. 2, an example is provided for further illustrating the signal processing operations of an ACO-OFDM transmitter. In the example given, the system has a total of 16 subcarriers, hence capable of conveying 4 symbols per OFDM block.

Fig. 2a shows the FD representation of the signal $\bar{S}$. It becomes clear that the requirements of Hermitian symmetry and blank subcarrier between every pair of subcarriers specified by (1) are strictly followed. As a result, the TD counterpart $\bar{s}$ of the FD signal $\bar{S}$ behaves exactly as portrayed in (2). In Fig. 2b, example data samples are provided to verify this property at a glance. Bear in mind that all TD samples in Fig. $2 \mathrm{~b}$ are real-valued. The TD and FD views of the clipped signal $s$ are provided in Figs. 2c and 2d, respectively. It can be seen from Fig. 2d that all clipping distortions fall on the subcarriers having even indices. The associated mathematical proof can be found in Appendix A.

3) ACO-OFDM Receiver: Fig. 3 shows the receiver schematic of ACO-OFDM.

The FD signal $\bar{R}$ is firstly obtained by passing the received TD signal $\boldsymbol{r}$ through $K$-point FFT. Based on the previous

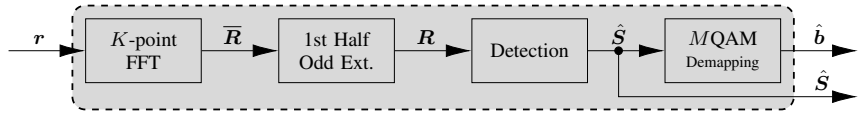

Fig. 3. Receiver block of DCO-OFDM (ACO RX).

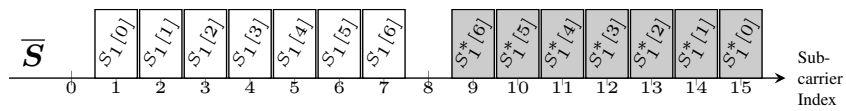

(a) Loaded FD Signal

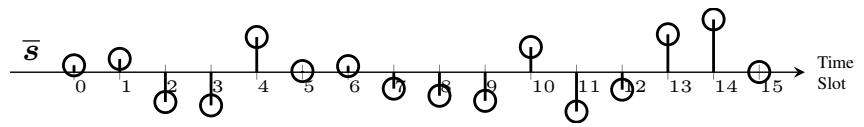

(b) Loaded TD Signal

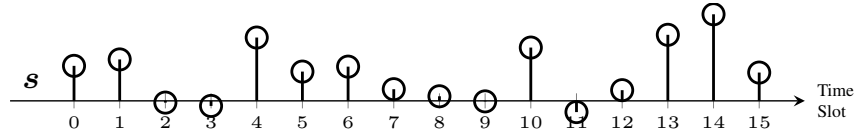

(c) DC-Biased TD Signal

Fig. 4. Example of DCO-OFDM TX process signal flow.

analysis, the negative-valued samples' clipping distortion only falls on the even-indexed subcarriers $(k=2 u)$ left blank at the loading stage, hence the message $\boldsymbol{R}$ of Fig. 3 can be extracted from the first half of the odd-indexed subcarriers, yielding:

$$
R[u]=\bar{R}[2 u+1], \quad 0 \leq u \leq\left(\frac{K}{4}-1\right) .
$$

The information symbols $\hat{\boldsymbol{S}}$ of Fig. 3 are then detected from the symbols $\boldsymbol{R}$ based on the constellation $\mathcal{S}$, which is formulated as

$$
\hat{S}[u]=\arg \min _{X \in \mathcal{S}}|X-2 \bar{R}[u]|, \quad 0 \leq u \leq\left(\frac{K}{4}-1\right),
$$

where the multiplicative factor of 2 for $\overline{\boldsymbol{R}}$ compensates the signal power halving during in the clipping process. Finally, the bit stream $\hat{b}$ is obtained at the receiver by mapping the symbols $\hat{\boldsymbol{S}}$ back to bits, as seen in Fig. 3 .

It is worth noting that the ACO RX block of Fig. 3 has both $\hat{\boldsymbol{b}}$ and $\hat{\boldsymbol{S}}$ as its output, where the former is the direct detection output, while the latter will be described in the next section on LACO-OFDM detection.

\section{B. Direct-Current-biased Optical OFDM}

Here, we also briefly review the processing and detection of DCO-OFDM signals, which is depicted in Fig. 4.

Similar to the signal generation of ACO-OFDM, Hermitian symmetry is also required in DCO-OFDM for obtaining a realvalued TD signal after IFFT. On the other hand, in DCOOFDM, there is no blank subcarrier. Therefore, the negative samples in the TD signal cannot be simply clipped, because they contain valuable message-related information. Instead, a DC component is added to the signal for shifting it into the positive domain, so that all TD samples become positivevalued for optical transmission. In this way, the bandwidthefficiency of DCO-OFDM becomes twice that of ACOOFDM, albeit the cost of consuming additional DC power. 
TABLE V

PROS AND CONS OF DCO- AND ACO-OFDM SCHEMES

\begin{tabular}{c|l|l}
\hline Scheme & \multicolumn{1}{|c|}{ Power Domain } & \multicolumn{1}{|c}{ Spectrum Domain } \\
\hline DCO-OFDM & $\begin{array}{l}\text { Less efficient due to } \\
\text { DC offset }\end{array}$ & $\begin{array}{l}\text { Half of the subcar- } \\
\text { riers are information- } \\
\text { bearing }\end{array}$ \\
\hline ACO-OFDM & $\begin{array}{l}\text { Efficient thanks to the } \\
\text { absence of DC offset }\end{array}$ & $\begin{array}{l}\text { A quarter of the } \\
\text { subcarriers are } \\
\text { information-bearing }\end{array}$ \\
\hline
\end{tabular}

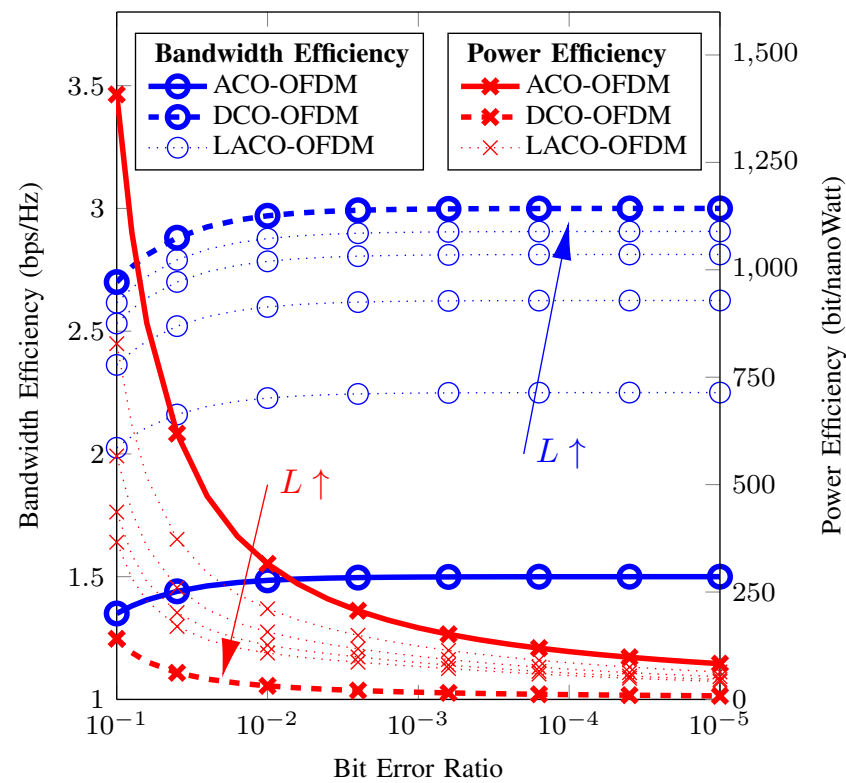

Fig. 5. Comparison of bandwidth and power efficiency for ACO-OFDM (solid lines) and DCO-OFDM (dashed lines). Blue lines marked by circles and scaled on the left axis represent the bandwidth efficiency, while those in red, marked by crosses and scaled on the right axis represent power efficiency. In addition, some dotted lines are depicted to indicate possible trade-offs, which will be introduced in the next section as LACO-OFDM schemes. Following the arrows of the relevant color, the dashed lines present LACO-OFDM of 2 , 3, 4 and 5 layers. The data are obtained for 64QAM modulation and the DC offset for DCO-OFDM is $13 \mathrm{~dB}$ based on [19]. The definitions of $\mathrm{BE}$ and $\mathrm{PE}$ in this figure are given in Appendix B.

\section{Power- vs. Bandwidth-Efficiency for ACO- and DCO- $O F D M$}

Tab. V summarizes the pros and cons of DCO-OFDM and ACO-OFDM, where a clear trade-off can be found between their BE and PE. More explicitly, Fig. 5 qualitatively portrays the SE and PE of the two schemes under same bandwidth and modulation constellation (64QAM), as they change with the improvement of system BER. Observe from Fig. 5 that huge gaps exist between DCO-OFDM and ACO-OFDM, and it would be beneficial if some other schemes can be provided to strike a better trade-off between SE and PE. In the next section, we will discuss LACO-OFDM as one of the most recent proposed candidate to solve this issue.

\section{Section Summary}

In this section, we reviewed the classic O-OFDM schemes ACO-OFDM and DCO-OFDM schemes. Their BE and PE performances were discussed, inspiring the conception of a

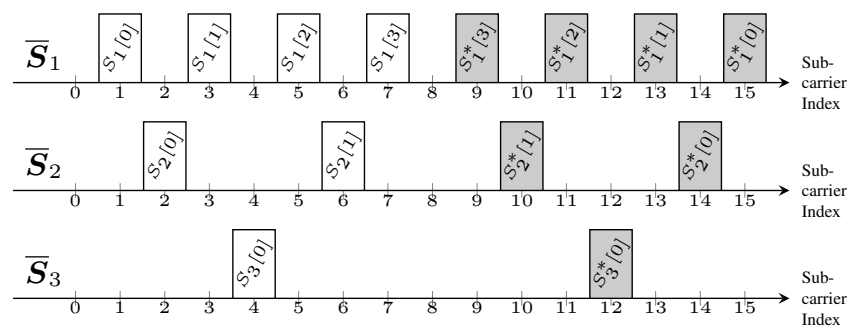

(a) FD

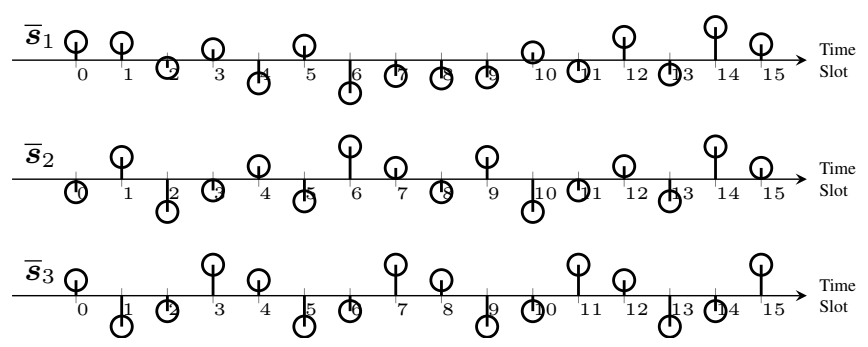

(b) TD

Fig. 6. Example of 3-layer 16-subcarrier LACO-OFDM TX process for mapping symbols on each layer, viewed at both FD and TD.

more flexible O-OFDM scheme. In the next section, we will discuss LACO-OFDM as one of the most recently proposed candidate for solving this challenge.

\section{LACO-OFDM TRANSMITTER}

In this section, we first analyze the composition of the LACO-OFDM signal and consider a pair of transmitter designs, discussing their pros and cons, followed by the characterization of LACO-OFDM signals, including their PDF and PAPR.

Again, LACO-OFDM actively exploits the even-indexed subcarriers $(k=2 u)$ of the ACO-OFDM frame, which were originally loaded with null symbols for accommodating the clipping distortion. An additional layer of ACO-OFDM symbols having half the length can then be transmitted, since the known clipping distortion can be readily canceled out at the receiver. More specifically, an ACO-OFDM scheme relying on $K=K_{1}$-point IFFT constitutes the first layer $(l=1$, referred to as 'layer 1') of a LACO-OFDM signal. Then, the $K_{2}=K_{1} / 2=K / 2$ even-indexed subcarriers are filled by the second layer ( $l=2$,referred to as 'layer 2 '), relying on $K_{2}$-point IFFT. Similarly, additional ACO-OFDM layers can be harnessed, so that the number of subcarriers occupied by layer $l$ becomes

$$
K_{l}=\frac{K_{l-1}}{2}=\frac{K}{2^{l-1}} .
$$

\section{A. Transmitter Signal Flow}

1) Mapping the Symbols to the L Layers: To begin with, the FD symbol stream $S$ generated from the $M$ QAM symbol set $\mathcal{S}$ is converted into $L$ parallel streams, which are mapped to each of the $L$ layers. For layer 1, ACO-OFDM relying on $K$-point IFFT is applied, which conveys a total of $K / 4$ QAM symbols. According to Section II-A, $K / 2$ subcarriers will be 


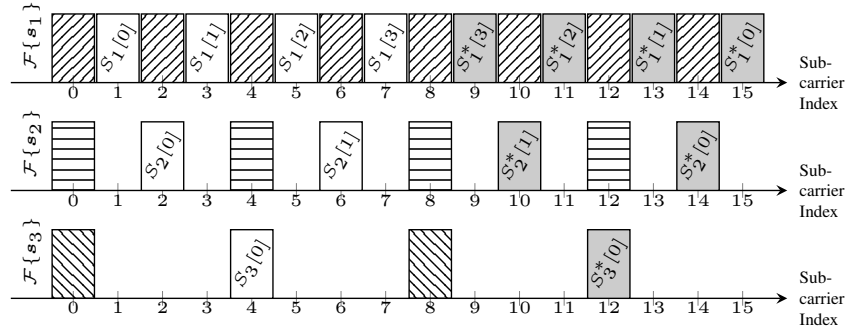

(a) FD

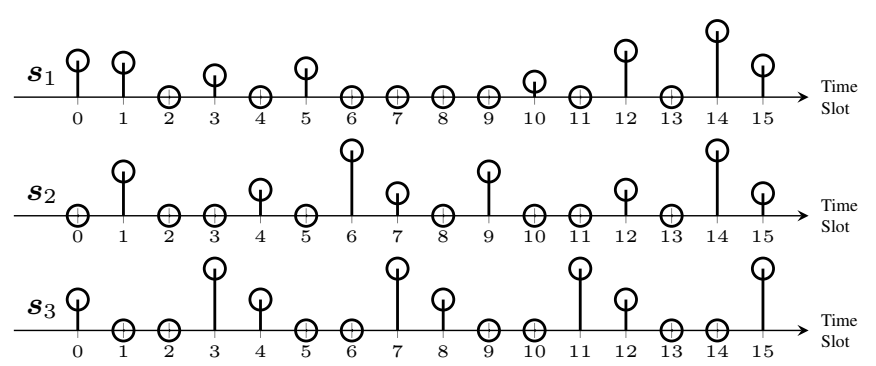

(b) TD

Fig. 7. Example of 3-layer 16-subcarrier LACO-OFDM TX process for clipping signals on each layer, viewed at both FD and TD.

left blank, which can then be utilized by the ACO-OFDM scheme on layer 2, relying on the halved IFFT length and conveying a halved number of symbols with respect to layer 1. The same arrangement is then applied to all layers, until all $L$ layers are filled with useful payload. As seen in Fig. 6a, the symbols on each layers $\left(\boldsymbol{S}_{l}\right)$ and their conjugates $\left(\boldsymbol{S}_{l}^{*}\right.$, shown as shaded bricks) are aligned with their corresponding FD subcarriers, while the unused FD positions are left zero.

For each layer, the signal $\overline{\boldsymbol{S}}_{l}$ is converted by the $K$-point IFFT into the TD signal $\overline{\boldsymbol{s}}_{l}$, which is real-valued and antisymmetric, as described by (2) and shown in Fig. 6b. As shown in Fig. $6 \mathrm{~b}$, the first half of $\overline{\boldsymbol{s}}_{2}$ at indices $(0, \ldots, 7)$ repeats itself at indices $(8, \ldots, 15)$. Similarly, the first quarter of $\bar{s}_{3}$ repeats at indices $(0, \ldots, 3)$ repeats itself three times.

2) Clipped Signals on each Layer: Then, as seen in Fig. 7b, lower boundary (LB) clipping is applied to these TD signals, which removes all negative time samples and produces the non-negative ACO-OFDM signal $s_{l}$. As discussed in Section II-A2, the clipping distortions fall on the empty subcarriers, as shown in Fig. 7a using diagonal and vertical patterns. Explicitly, the clipping distortions of layer 1 fall on all evenindexed subcarriers, while those of layer 2 on subcarriers 0 , 4,8 and 12 , and finally those of layer 3 on subcarriers 0 and 8.

3) Superimposed LACO-OFDM Signal: Next, all nonnegative layer signals are superimposed on each other, in order to form the signal $\boldsymbol{x}$. As shown in Fig. 8a, all the symbols of layer 1 occupy their own subcarriers, while those of layer 2 must share their subcarrier with the clipping distortions of layer 1 . Moreover, the two subcarriers accommodating layer 3 symbols, i.e. subcarriers 4 and 12, also host the clipping distortions of layers 1 and 2.

$\boldsymbol{x}$ is then transmitted over the optical IM/DD channel, and would be received by the RX with the aid of a PD.

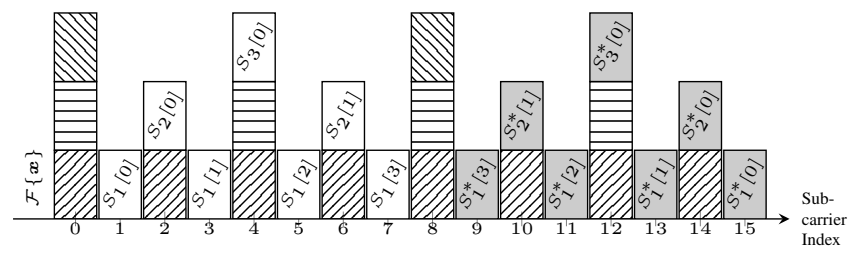

(a) FD

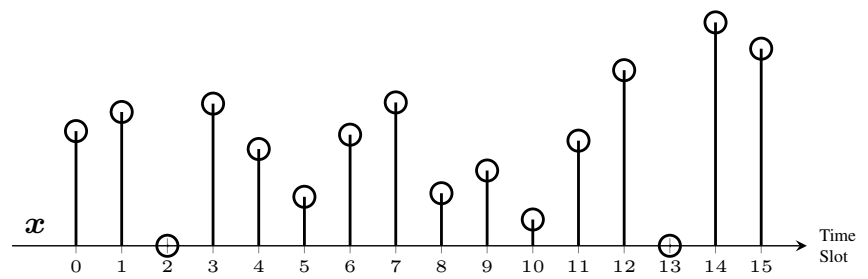

(b) TD

Fig. 8. Example of 3-layer 16-subcarrier LACO-OFDM TX process for superimposing layered signals together, viewed at both FD and TD.

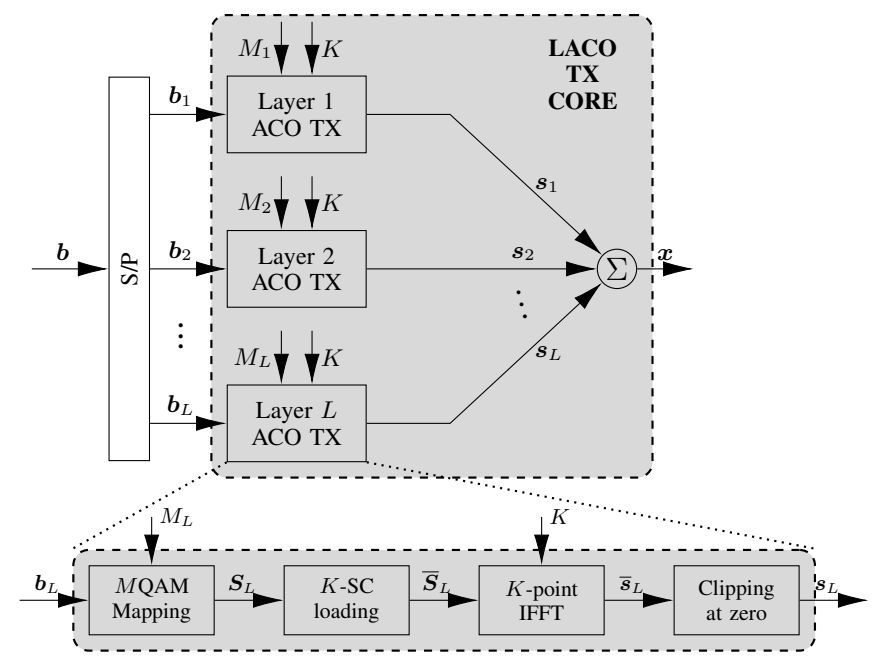

Fig. 9. LACO-OFDM transmitter system model 1, where the zoom-in structure of ACO TX similar to Fig. 1 is provided.

4) Further Clipping of the Signal: In reality, the intensity modulated optical signal would also be restricted by the hardware's (e.g. LED) limited linear range, as well as by the maximum instantaneous power limit. Hence, $\boldsymbol{x}$ will be further clipped at the upper bound (UB) $B_{\mathrm{u}}$, resulting in the upper-bounded signal $\lceil\boldsymbol{x}\rceil$. Hence, we have $\lceil x\rceil[k]=$ $\left\{\begin{array}{l}x[k], x[k]<B_{\mathrm{u}} \\ B_{\mathrm{u}}, \text { otherwise. }\end{array}\right.$

\section{B. LACO-OFDM Transmitter Designs}

Below we present a pair of LACO-OFDM transmitters found in the literature, before discussing their pros and cons.

1) Model 1: Fully-Parallel Design: Fig. 9 shows the block diagram of an $L$-layer LACO-OFDM transmitter, where the $L$ bit streams after $\mathrm{S} / \mathrm{P}$ are fed into their respective ACOOFDM TX blocks. Observe from Fig. 9 that all ACO-OFDM components rely on $K$-point IFFT. 


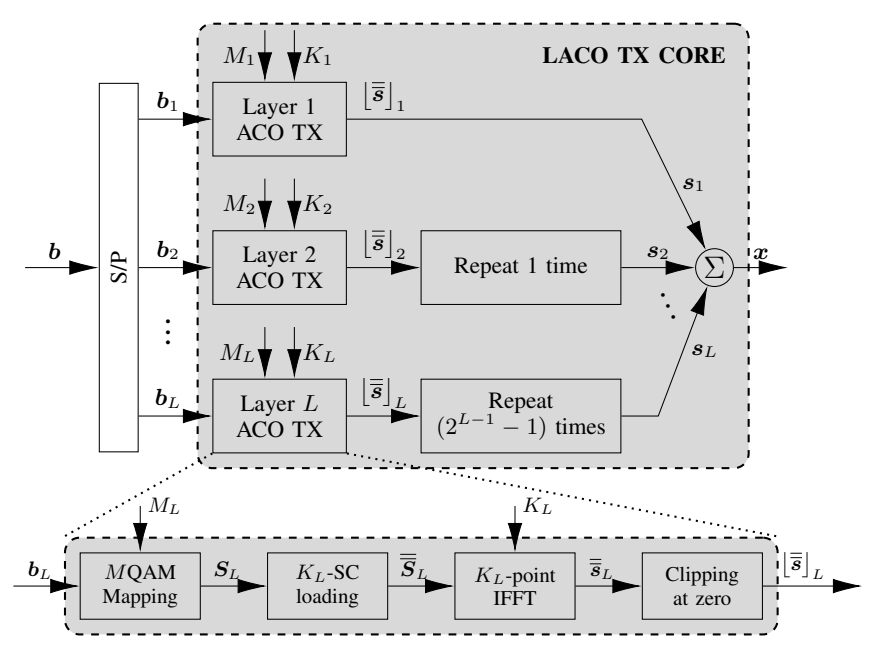

Fig. 10. LACO-OFDM transmitter system model 2, where the zoom-in structure of ACO TX similar to Fig. 1 is provided.

The bit-to-symbol mapping and symbol-to-subcarrier loading strategies of layer 1 is the same as that of ACO-OFDM in (1), while for higher layers this should be modified. Since layer 2 occupies all the even-indexed subcarriers, i.e. $k=2,4,6,8, \ldots$, to obey the rule of ACO-OFDM by leaving every other subcarrier blank, the symbols of layer 2 can only be loaded onto those subcarriers whose indices are multiples of 4 , i.e. $k=4,8,16, \ldots$, leaving the rest blank. Similarly, layer 3 occupies subcarriers $k=2,6,10,14, \ldots$ and only load symbols on $k=6,14,22, \ldots$ This strategy is summarized in (6), where for layer $l$ the symbols are loaded onto subcarriers having indices, which are multiples of $2^{l}$ shifted by an additional $2^{l-1}$ positions, and the conjugated symbols are followed in a descending order

$$
\begin{gathered}
\bar{S}_{l}[k]=\left\{\begin{array}{l}
S_{l}[u], \quad k=2^{l} u+2^{l-1}, \\
S_{l}^{*}[u], \quad k=K-\left(2^{l} u+2^{l-1}\right), \\
0, \quad \text { otherwise },
\end{array}\right. \\
1 \leq l \leq L, 0 \leq u \leq \frac{K}{2^{l+1}} \text { for layer } l, 0 \leq k \leq K-1,
\end{gathered}
$$

Each of these loaded FD symbol streams $\overline{\boldsymbol{S}}_{l}$ of Fig. 9 is independently converted into a TD signal $\bar{s}_{l}$ formulated as:

$$
\overline{\boldsymbol{s}}_{l}=\mathcal{F}^{-1}\left\{\overline{\boldsymbol{S}}_{l}\right\}
$$

while have their negative samples clipped as follows:

$$
\boldsymbol{s}_{l}=\left\lfloor\overline{\boldsymbol{s}}_{l}\right\rfloor \text {. }
$$

The resultant unipolar signals $s_{l}$ are finally superimposed to form the LACO-OFDM signal $\boldsymbol{x}$ of Fig. 9, which will be forwarded to the D/A process.

2) Model 2: Minimum-Cost Design: Fig. 10 provides another portrayal of the LACO-OFDM transmitter, where signal repetition is required for the higher layers, in exchange for having a reduced number of IFFT stages. In the design shown in Fig. 10, the symbols on all layers are loaded onto subcarriers in the same manner as in ACO-OFDM, leaving only one blank subcarrier between each active pair. Since the number of subcarrier symbols conveyed by each layer is different, the lengths of the loaded signals $\overline{\bar{S}}_{l}$ of Fig. 10 also vary. Explicitly, we have:

$$
\begin{gathered}
\overline{\bar{S}}_{l}[k]=\left\{\begin{array}{cc}
S_{l}[u], & k=2 u+1, \\
S_{l}^{*}[u], & k=K_{l}-(2 u+1), \\
0, & \text { otherwise },
\end{array}\right. \\
1 \leq l \leq L, 0 \leq u \leq \frac{K}{2^{l+1}} \text { for layer } l, 0 \leq k \leq K_{l}-1 .
\end{gathered}
$$

This in turn implies that the TD signal must repeat itself several times for $l>1$ for the sake of appropriately aligning the TD signals of all the $L$ layers. Since for the higher layers $(l \geq 2)$, the IFFT produce TD signals shorter than $K$ samples, given the reduced-length IFFT stages, repetition is required for appropriately aligning them with the length- $K$ layer 1 signal. Then, the attenuation factor of $\sqrt{K_{k} / K}$ has to be applied after repetition for energy conservation, so that the expanded signal $s_{l}$ has the same energy as $\overline{\bar{s}}_{l}$, yielding:

$$
s_{l}[n]=\sqrt{\frac{K_{l}}{K}}\lfloor\overline{\bar{s}}\rfloor\left[\bmod \left(n, K_{l}\right)\right] .
$$

Finally, following superposition, the LACO-OFDM signal $\boldsymbol{x}$ of Fig. 10 is now ready for electric/optical conversion.

3) Discussions: Despite the fact that the two TX models produce exactly the same LACO-OFDM signals, in practice, more detailed issues have to be discussed in terms of their implementation.

Model 1 represents a balanced design, where the data processing of all layers follow the same structure. Therefore, the same layer processing module can be reused for all layers, hence potentially reducing the complexity. For a standard length- $K$ IFFT operation, where $K$ is a power of 2, the number of Complex Multiplications and Additions (CMA) equals $K \log _{2} K$. Therefore, a LACO-OFDM system having $L$ layers requires a total of $2 L K \log _{2} K$ CMAs using Model 1. Since the data processing of each layer can be operated independently, a parallel structure can be employed for potentially increasing the bit rate at a given processing power. If the time delay of a single IFFT stage is $T_{\mathrm{IFFT}}$, the total time required for Model 1 will be proportional to $T_{\mathrm{IFFT}} \log _{2} K$.

On the other hand, TX Model 2 reduces the IFFT complexity by inserting fewer zeros at the symbol-to-subcarrier loading stage, resulting in shorter $\overline{\bar{S}}_{l}$ sequences. The number of IFFT points for layer $l$ is $K_{l}=K / 2^{l-1}$ according to (5), yielding a total of $\frac{2 K}{2^{l-1}} \log _{2} \frac{K}{2^{l-1}}$ CMAs. Therefore, the total number of CMAs for a LACO-OFDM system having $L$ layers using TX Model 2 will be

$$
\begin{aligned}
& \sum_{l=1}^{L}\left(\frac{2 K}{2^{l-1}} \log _{2} \frac{K}{2^{l-1}}\right) \\
= & 2 K \log _{2} K \sum_{l=1}^{L} \frac{1}{2^{l-1}}-2 K \sum_{l=1}^{L} \frac{l-1}{2^{l-1}} \\
= & 4\left(1-\frac{1}{2^{L}}\right) K \log _{2} K-\frac{2 K}{2^{L-1}}\left(2^{L}-L-1\right) \\
< & 2 K \log _{2} K, \forall l>1 .
\end{aligned}
$$

The reduced IFFT complexity comes at the cost of repeating higher layer TD signals multiple times for aligning them with the length- $K$ layer 1 . However, the repetition operation can be 


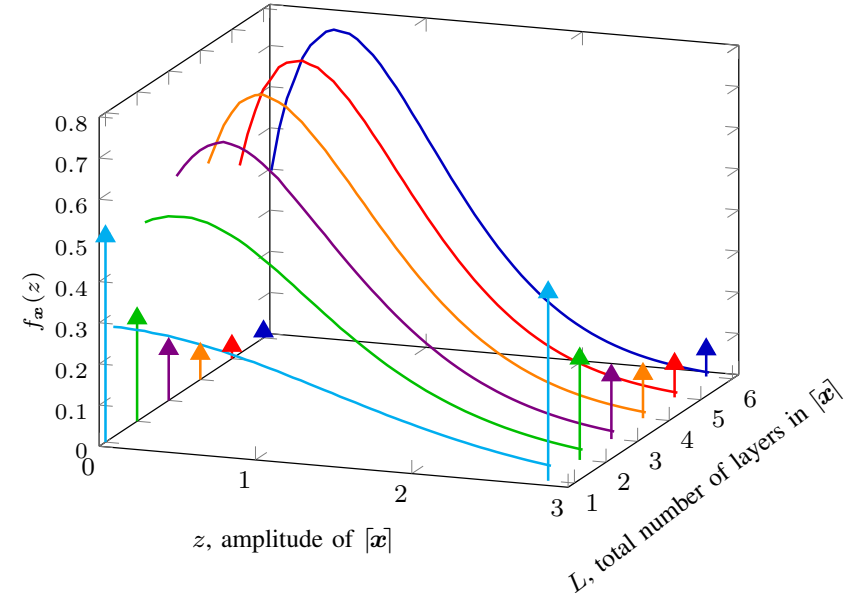

Fig. 11. PDF of UB-clipped LACO-OFDM signal $\lceil\boldsymbol{x}\rceil$ at an upper boundary $B_{\mathrm{u}}=2.818 \mathrm{~V}$. The impulses at $z=B_{\mathrm{u}}$ have been amplified 20 times for a better legibility.

carried out while waiting for the lower layers' IFFT processes to be completed, which are more time consuming due to having more stages included. Therefore, the total time delay of the Model 2 IFFT is dominated by its layer 1 process, which is the same as in model 1, i.e. $T_{\mathrm{IFFT}} \log _{2} K$.

The discussions above are summarized below in Tab. VI. Note that the number of CMAs is estimated based on the classic RF-OFDM system. Since only a fraction of subcarriers are loaded with complex-valued symbols in O-OFDM, this number can be reduced by carefully optimizing the IFFT structures. Nevertheless, the IFFT complexity of Model 1 will always be higher than that of Model 2.

It is clear that Model 2 should be employed in practical design, as a benefit of its lower complexity. However, for the sake of analyzing the LACO-OFDM system, both models are used in this paper, but different notations are assigned to distinguish them.

\section{LACO-OFDM Signal Characterization}

Having outlined the process of generating the LACOOFDM signals at the transmitter, in this section we focus our attention on its characterization. Explicitly, we will characterize both the Probability Density Function (PDF) and PAPR of LACO-OFDM.

1) Probability Density Function: Before we delve into the analysis, let us first take a look at the PDF of LACOOFDM signals having 1 to 6 layers, with the UB set to $z=B_{\mathrm{u}}=2.818 \mathrm{~V}$, as presented in Fig. 11. The impulses representing the LB and UB clipping are also shown in Fig. 11, noting that the impulses representing the UB clipping at $z=B_{\mathrm{u}}$ are amplified by a factor of 20 for a better view of their relationships. Observe from Fig. 11 that

(a) while the PDF of single-layer LACO-OFDM is a truncated one-sided Gaussian distribution, the peaks gradually move towards higher values of $z$, as more layers are added, while the mean is increased and the variance is reduced, as total number of layers $L$ is increased;

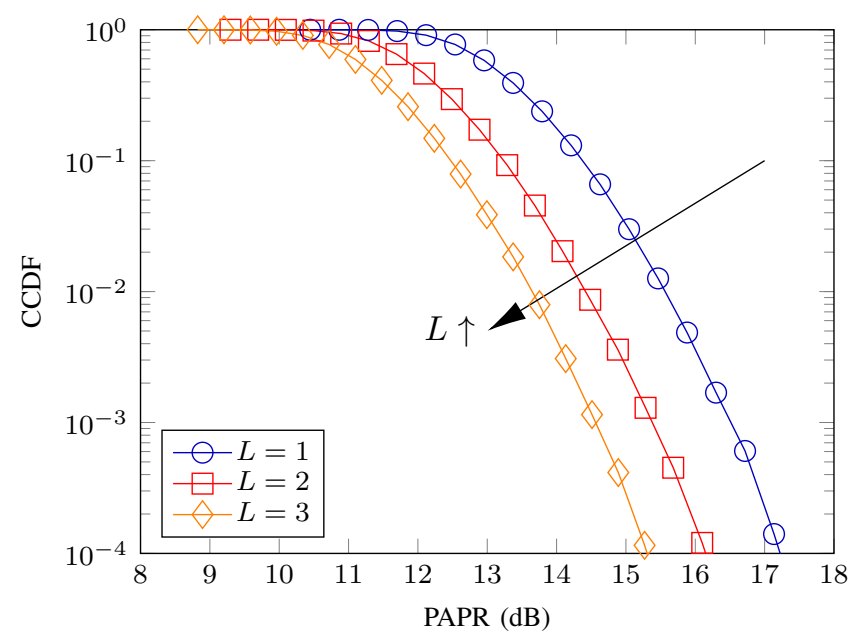

Fig. 12. CCDF of the PAPR of 1024-subcarrier LACO-OFDM TD signals.

(b) the signals having more layers statistically speaking are more likely to have lower amplitude, which influences their respective PAPR;

(c) the single-layer LACO-OFDM, namely the ACO-OFDM system, has the highest UB clipping distortion, as indicated by its highest impulse at $z=B_{\mathrm{u}}$.

2) Peak-to-Average Power Ratio: Fig. 12 compares the Complementary Cumulative Distribution Function (CCDF) of the PAPR of LACO-OFDM signals having different number of layers $L$, where the number of subcarriers $K$ is fixed to 1024. It can be seen that LACO-OFDM having more layers (i.e. higher $L$ ) is more likely to have lower PAPR, which in turn indicates that the TD signal amplitude becomes more flat, as and when more layers are superimposed.

\section{Section Summary}

In this section, we introduced the transmitter of LACOOFDM. We presented the signal flow with the aid of lowpaced examples of the transmitter processing operations and presented two different designs for the transmitter. We then studied the PDF and PAPR performance of LACO-OFDM signals, showing that increasing the number of layers reduces the PAPR. In the next section, we will then take a look at the receiver of LACO-OFDM.

\section{LACO-OFDM RECEIVER}

Having covered the composition of the LACO-OFDM signal in the previous section, here we study its receiver design. Explicitly, we present its iterative detection procedure, with the aid of detailed TD and FD examples similar to the style of the previous section. Its BER performance is also studied.

\section{A. Received LACO-OFDM Signal}

Let us first consider the received signal. 
TABLE VI

COMPARISON OF THE TWO TRANSMITTER DESIGNS IN LACO-OFDM

\begin{tabular}{cccl}
\hline Model & CMA & Delay & \multicolumn{1}{c}{ Comment } \\
\hline Model 1 & $2 K \log _{2} K$ & $T_{\text {IFFT }} \log _{2} K$ & Balanced design \\
Model 2 & $4\left(1-\frac{1}{2^{L}}\right) K \log _{2} K-\frac{2 K}{2^{L-1}}\left(2^{L}-L-1\right)$ & $T_{\text {IFFT }} \log _{2} K$ & Less IFFT complexity \\
\hline
\end{tabular}

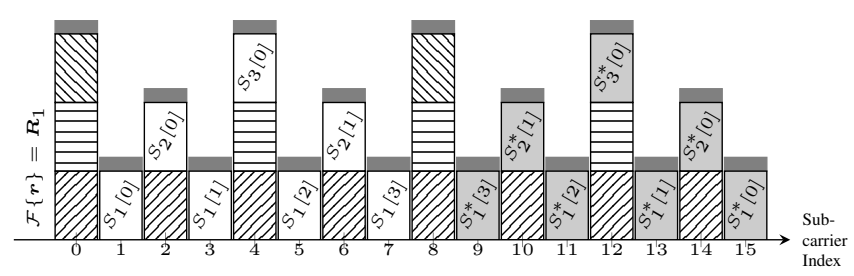

(a) $\mathrm{FD}$

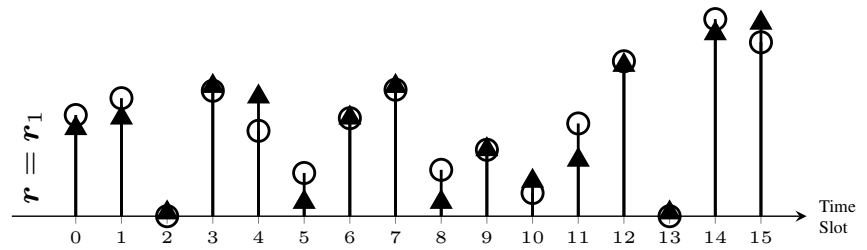

(b) TD

Fig. 13. Example of 3-layer 16-subcarrier LACO-OFDM received signal, viewed at both FD and TD.

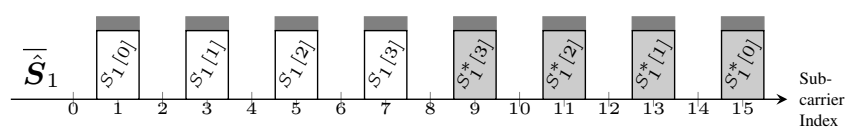

(a) FD

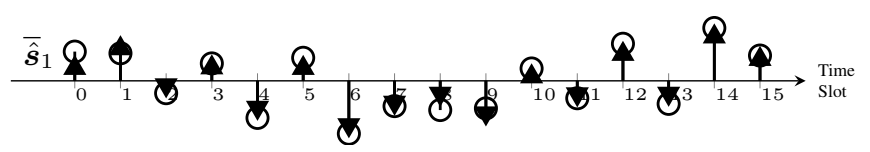

(b) TD

Fig. 14. Example of 3-layer 16-subcarrier LACO-OFDM RX process for detecting layer 1, viewed at both FD and TD.

1) Frequency Domain Composition of Received Signal:

Due to the noisy optical channel, the received signal $r$ becomes slightly different from the transmitted signal $x$. In Fig. 13b, the received signal is shown in triangular stems, while the original transmitted signal $\boldsymbol{x}$ is represented by circular stems for comparison.

Meanwhile, layer 1 is free from any clipping distortions based on our previous analysis. Therefore, at this stage $(l=1)$ the clipping distortion subtraction operation does not affect the signal, and $\boldsymbol{r}$ passes as $\boldsymbol{r}_{1}$ to the next step, yielding $\boldsymbol{r}=\boldsymbol{r}_{1}$.

First, $\boldsymbol{r}_{1}$ is converted by $K$-point FFT into its FD counterpart $\overline{\boldsymbol{R}}_{1}$, where the message symbols of all $L$ layers, their corresponding clipping distortions and the noise co-exist, as shown in Fig. 13a. It is worth mentioning that as the thermal noise is Gaussian, it spreads evenly across the full frequency spectrum, which is reflected in Fig. 13a as the tiny dark gray bricks on top of all the subcarriers.

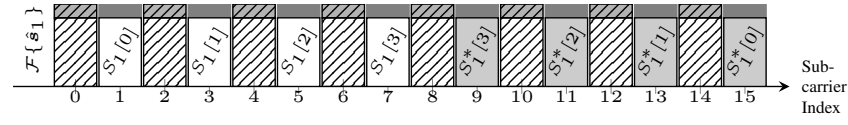

(a) FD

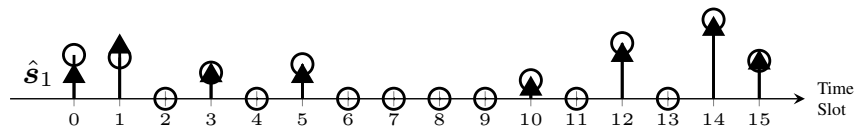

(b) $\mathrm{TD}$

Fig. 15. Example of 3-layer 16-subcarrier LACO-OFDM RX process for regenerating the interference of layer 1, viewed at both FD and TD.

2) Layer 1 Signal Detection: Iterative detection is then carried out, where layer $l$ is detected in the $l$ th iteration. Since layer 1 suffers from no clipping distortion contamination, its symbols can be directly extracted from $\overline{\boldsymbol{R}}_{1}$ as $\boldsymbol{R}_{1}$, which is shown in Fig. 14 and formulated as $R_{1}[u]=\bar{R}_{1}[2 u+1]$. This is the inverse operation of (6) for $l=1$. Based on the $M_{1} \mathrm{QAM}$ mapping constellation $\mathcal{S}_{1}$, the estimated transmitted symbols $\hat{\boldsymbol{S}}_{1}$ on layer 1 can be detected as

$$
\hat{S}_{1}[u]=\arg \min _{X \in \mathcal{S}_{1}}\left|X-2 R_{1}[u]\right|, \quad 0 \leq u \leq\left(\frac{K_{1}}{4}-1\right) .
$$

\section{B. Serial Interference Cancellation for Higher Layer Detec- tion}

1) Layer 1 Clipping Distortion Regeneration and Cancellation: Then, the same ACO-OFDM TX processing as mentioned in Section II-A1 is applied to $\overline{\hat{S}}_{1}$, producing the TD signal $\overline{\hat{s}}_{1}$ after IFFT and $\hat{s}_{1}$ after UB clipping, both shown in Fig. 15b. Due to the unavoidable thermal noise, $\overline{\hat{s}}_{1}$ would differ from $\bar{s}_{1}$ slightly, as seen in Fig. $15 \mathrm{~b}$, where the original signal $\bar{s}_{1}$ is marked by circles, and the locally generated $\overline{\hat{s}}_{1}$ by triangles. Viewing now these operations in the FD, the clipping distortion of layer 1 has now been re-generated at the RX as part of $\hat{s}_{1}$, apart from the slight difference from the actual distortion due to the noise, as shown in Fig. 15a, where this difference is represented by the small light gray bricks at the top of the clipping distortion bricks constituted by the even-index bars marked by the North East hatching pattern.

Since we are going to cancel the clipping distortion from the received signal, in order to detect the layer 2's signal, the additional light gray bricks will interfere with the detection process and degrade the performance. Since this interference arises from layer 1 and affects the performance of layer 2, we refer to it as the Inter-Layer Interference (ILI) imposed by layer 1 . 


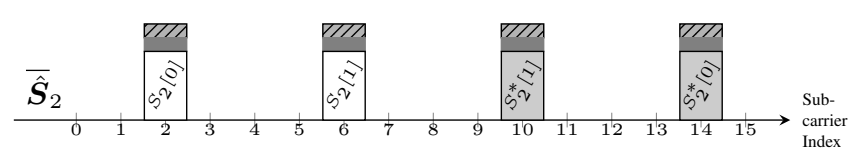

(a) FD

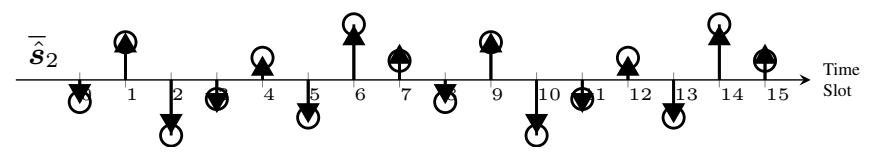

(b) $\mathrm{TD}$

Fig. 16. Example of 3-layer 16-subcarrier LACO-OFDM RX process for detecting layer 2, viewed at both FD and TD.

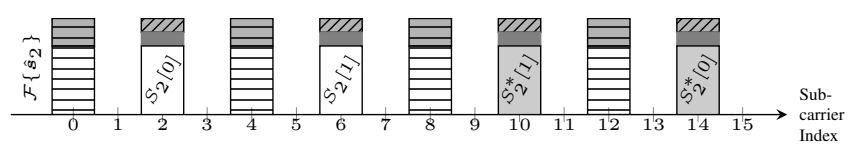

(a) $\mathrm{FD}$

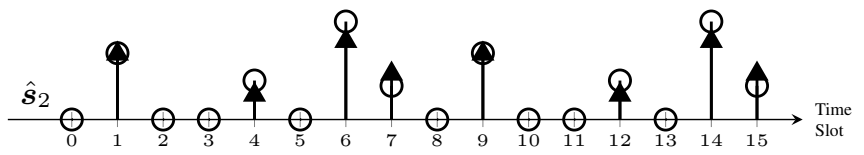

(b) $\mathrm{TD}$

Fig. 17. Example of 3-layer 16-subcarrier LACO-OFDM RX process for regenerating the interference of layer 2, viewed at both FD and TD.

2) Layer 2 Signal Detection: In the second stage of the RX processing, layer 2 has to be detected. The locally generated layer 1 signal $\hat{s}_{1}$ constituted by the even-index bars marked by the North East hatching pattern is subtracted from $\boldsymbol{r}_{1}$ for canceling layer 1's clipping distortion. Therefore, in the resultant $\boldsymbol{r}_{2}$, the symbols of layer 2 are decontaminated and can be directly detected.

Likewise, $\boldsymbol{r}_{2}$ is transformed by a $K$-point FFT to form $\boldsymbol{R}_{2}$. The symbols of layer 2 can then be extracted from $\overline{\boldsymbol{R}}_{2}$ as $\boldsymbol{R}_{2}$, yielding:

$$
R_{2}[u]=\bar{R}_{2}[4 u+2]=\bar{R}_{1}[4 u+2]-\mathcal{F}\left\{\hat{\boldsymbol{s}}_{1}\right\}[4 u+2],
$$

where the ' $4 u+2$ ' indexing comes from the relationship of ' $k=2^{l} u+2^{l-1}$ ' in (6).

Then, $\hat{\boldsymbol{S}}_{2}$ is assembled into the length- $K$ signal $\overline{\hat{\boldsymbol{S}}}_{2}$ as in (6), as shown in Fig. 17a. Upon comparing Fig. 17a and Fig. 15a, it becomes clear that the receiver's copy of the layer 2 signal is contaminated not only by the noise, but also by the ILI of layer 1 . The detected layer 2 symbols are then passed through the ACO-OFDM TX process for regenerating its clipping distortion at the RX, hence facilitating the decontamination and detection of layer 3 , using the same procedure as mentioned above. The RX keeps on repeating the detection-regeneration-cancellation loop, until all $L$ layers become detected.

\section{Receiver Design}

Fig. 18 shows the block diagram of a basic iterative LACOOFDM receiver invoking the detection method detailed in Sections IV-A and IV-B. The "ACO RX" module of Fig. 3

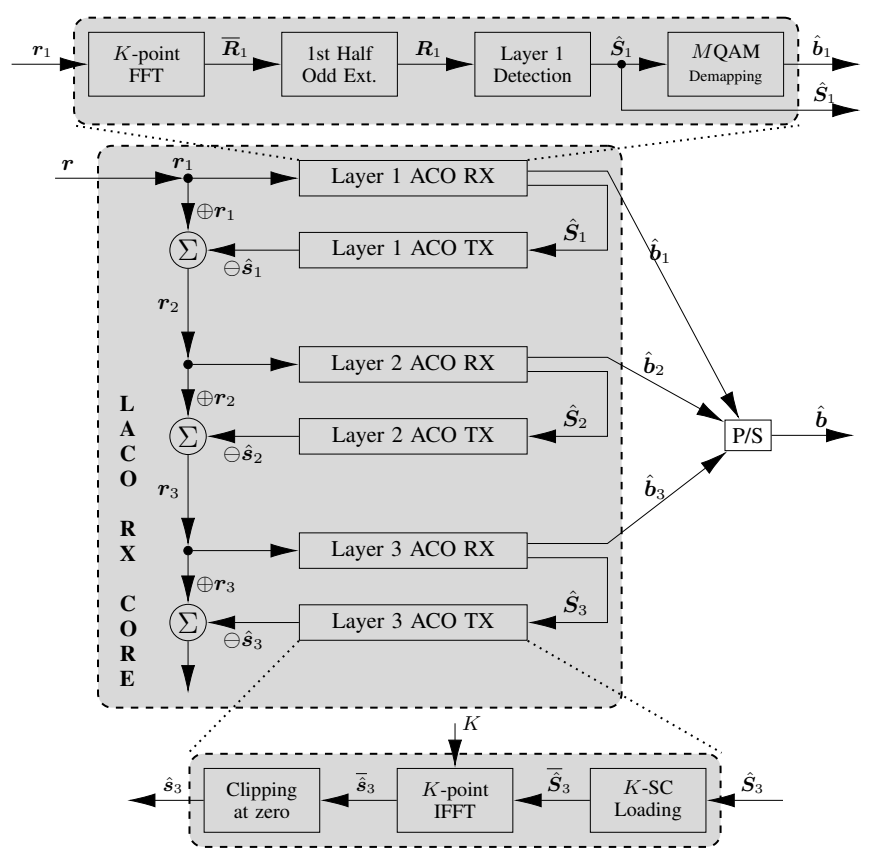

Fig. 18. LACO-OFDM receiver system, where ACO TX and ACO RX represent the blocks seen in Figs. 1 and 3, respectively, and $\Sigma$ subtracts the signal marked with $\ominus$ from the signal marked with $\oplus$.

and the "ACO TX" module of Fig. 1 are cascaded for the detection-regeneration-cancellation loop of each layer.

For the detection of layer $l$, signal $\boldsymbol{r}_{l}$ having the clipping distortion of layers $1 \sim(l-1)$ removed $^{2}$ is then entered into the "Layer $l$ ACO RX" module for detection, where the detected bit stream $\hat{\boldsymbol{b}}_{l}$ and the symbol stream $\hat{\boldsymbol{S}}_{l}$ are obtained. $\hat{\boldsymbol{S}}_{l}$ is then forwarded to the "Layer $l$ ACO TX" module for regenerating the clipped signal $\hat{s}_{l}$ containing the clipping distortion of layer $l$, which will be subtracted from $\boldsymbol{r}_{l}$, in order to feed the detection-regeneration-cancellation loop of the next layer (layer $l+1$ ). Meanwhile, the streams $\hat{\boldsymbol{b}}_{l}$ of all layers $(l=1, \ldots, L)$ are collected, in order to reconstitute the single stream $\hat{b}$ of Fig. 18 to create the final output.

\section{Synchronization Issues}

Akin to its RF counter part, the BER performance of OOFDM can also be degraded due to synchronization errors, which has been studied for ACO-OFDM [75]. To elaborate a little further, the carrier frequency errors introduce intersubcarrier interference in the FD, while in the TD the misalignment of the FFT sampling window imposes phase error [76]. Therefore, accurate FD and TD synchronization must be maintained. Generally, the synchronization algorithms rely on an initial time and frequency acquisition stage, followed by a tracking stage that constantly monitors the slight deviations during each OFDM symbol, as detailed in [77] providing a rich survey of the associated techniques and their performance.

\footnotetext{
${ }^{2}$ When detecting layer 1 , we have simply $\boldsymbol{r}=\boldsymbol{r}_{1}$, since the layer 1 symbols are free from clipping distortion.
} 


\section{E. Performance Results and Discussions}

In this part, we are going to discuss the major factors influencing the BER performance of the LACO-OFDM receiver. We commence by considering the sources degrading the system performance, followed by our simulation results providing quantitative insights.

1) Sources of Performance Degradation: Apart from the ubiquitous AWGN, the received signal is contaminated by the ILI, as well as by the optical channel's clipping distortion.

a) Clipping: For signals transmitted through an IM/DD optical channel, amplitude clipping may occur. On the one hand, negative samples cannot be conveyed by intensity modulation, which are clipped during the processing of each layer. As discussed in Section III, this specific type of clipping does not $^{3}$ degrade the BER performance, which is a specific benefit of the LACO-OFDM. On the other hand, samples higher than the linear range of the transceiver will be clipped after the superposition of all layer signals. Due to the nonlinearity of this process, it is hard to mathematically quantify its effect. For 1-layer LACO-OFDM (i.e. ACO-OFDM), which is simply truncated-Gaussian-distributed, its clipping can be modeled according to Bussgang's theorem as an amplitude attenuation $\kappa$ of the signal $\boldsymbol{x}$ plus an uncorrelated additive zero-mean (but not necessarily white and Gaussian-distributed) noise $\boldsymbol{n}_{\mathrm{c}}$ [78], which is formulated as $\lceil\boldsymbol{x}\rceil=\kappa \boldsymbol{x}+\boldsymbol{n}_{\mathrm{c}}$. In this way, the effect of clipping would be similar to that of a noisy fading channel. For LACO-OFDM signals, which were shown to be non-Gaussian distributed in Fig. 11, the above-mentioned model can still be applied, but at slight loss of accuracy [45].

b) Inter-Layer Interference: To further elaborate on the effect of error propagation through the LACO-OFDM layers owing to the ILI, we provide examples of detecting subcarriers on the first, second and third layer of a LACO-OFDM in Fig. 19. Again, at the RX, layer 1's symbols are immune to ILI and can be detected directly, as it has been shown both in (12) and in Fig. 13a. If the instantaneous noise power is large enough to "push" a mapped symbol across a decision threshold, the symbol will be erroneously detected and the receiver would

(a) produce erroneously demapped bits for layer 1, directly increasing the BER, and

(b) regenerate erroneous clipping distortion, hence detrimentally affecting the detection of the following layers as well, which is detailed below.

As mentioned in Section IV-B2, the layer 2 signal is contaminated by both the noise and the ILI of layer 1, as depicted in Fig. 19b. This means that the misdetection of layer 2 symbols would have more severe effects on the BER than that of the layer 1 signals, due to the additional ILI.

More generally, the detection process for layer $l$ of the LACO-OFDM signal after the removal of the previous layers' clipping distortion is influenced not only by the thermal noise, but also by the ILI imposed by all detection errors occurring in layers $1 \sim(l-1)$. Therefore, a decision error, made when

\footnotetext{
${ }^{3}$ The error propagation and inter-layer interference that are indirectly related to the clipping is not considered here, but will be discussed separately in the next paragraph.
}

TABLE VII

INTERPRETATION OF THE COMPONENTS IN (16)

\begin{tabular}{cll}
\hline Label & \multicolumn{1}{c}{ Component } & \multicolumn{1}{c}{ Meaning } \\
\hline \multirow{2}{*}{$\mathrm{a}$} & $\left(\mathrm{a}_{1}\right) \kappa^{2}$ & UB clipping attenuation \\
\cline { 2 - 3 } & $\left(\mathrm{a}_{2}\right) \sigma_{\mathrm{c}}^{2}$ & UB clipping noise power \\
\hline $\mathrm{b}$ & $\gamma_{\mathrm{b}} N_{0}$ & Signal power per bit \\
\hline $\mathrm{c}$ & $\sum_{l=1}^{L}\left(2^{-l-1} \log _{2} M_{l}\right)$ & Overall bit rate \\
\hline $\mathrm{d}$ & $N_{0}$ & AWGN power \\
\hline $\mathrm{e}$ & $\sum_{i=1}^{l-1} \mathcal{G}_{i}$ & First $(l-1)$ layers' ILI [45] \\
\hline
\end{tabular}

detecting layer $l$, will increase the BER of both the current layer and that of the following layers (layers $(l+1) \sim L$ ), hence precipitating further performance degradation by ILI.

c) LACO-OFDM Bit Rate: As discussed in Section III-A, the number of subcarriers occupied by each layers varies, and so does their ability of conveying information bits. Therefore, the contribution of each layer to the overall BER is also different. For layer $l$, having a modulation order of $M_{l}$ and $\frac{K}{2^{l+1}}$ data-bearing subcarrier, a total of $\frac{K}{2^{l+1}} \log _{2} M_{l}$ bits can be transmitted. The bit rate of each layer also determines its share of error contribution towards the overall BER performance.

d) Overall BER Performance: The overall Error Probability (EP) $\mathbb{P}_{\mathrm{b}}$ of LACO-OFDM is given by the ratio of the sum of erroneous bits of all layers to the sum of transmitted bits of all layers. Physically, this represents the bit-rate-weighted average of the EP of each layer $\mathbb{P}_{\mathrm{b}, l}$ :

$$
\mathbb{P}_{\mathrm{b}}=\frac{1}{\sum_{l=1}^{L} 2^{-l} \log _{2} M_{l}} \sum_{l=1}^{L} 2^{-l} \mathbb{P}_{\mathrm{b}, l} \log _{2} M_{l} .
$$

The layer EP $\mathbb{P}_{\mathrm{b}, l}$ can be calculated in a similar way as in RF systems, i.e. based on the QAM order $M_{l}$ and the layer signal-to-noise-and-interference-plus-distortion ratio $\Gamma_{\mathrm{s}, l}$. Under the assumption of an AWGN channel, the layer EP can be expressed as [79]:

$$
\mathbb{P}_{\mathrm{b}, l} \approx \frac{4\left(\sqrt{M_{l}}-1\right)}{\sqrt{M_{l}} \cdot \log _{2} \sqrt{M_{l}}} \cdot Q\left(\sqrt{\frac{3 \Gamma_{\mathrm{s}, l} \log _{2} M_{l}}{M_{l}-1}}\right),
$$

where $Q(\cdot)$ is the tail probability of the normal distribution given by $Q(x)=\frac{1}{\sqrt{2 \pi}} \int_{x}^{\infty} \exp \left(-\frac{z^{2}}{2}\right) \mathrm{d} z$.

For higher layers $(l>1), \Gamma_{\mathrm{s}, l}$ can be expressed as

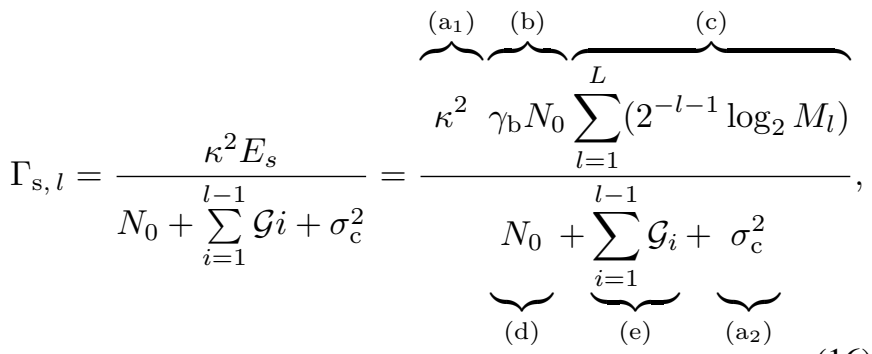

where the interpretation of the components in (16) is given below in Tab. VII. 


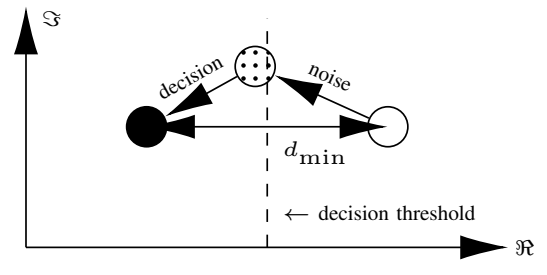

(a) Detecting Layer 1

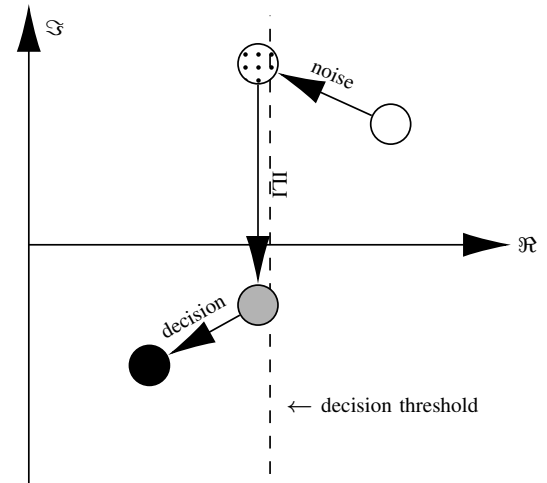

(b) Detecting Layer 2

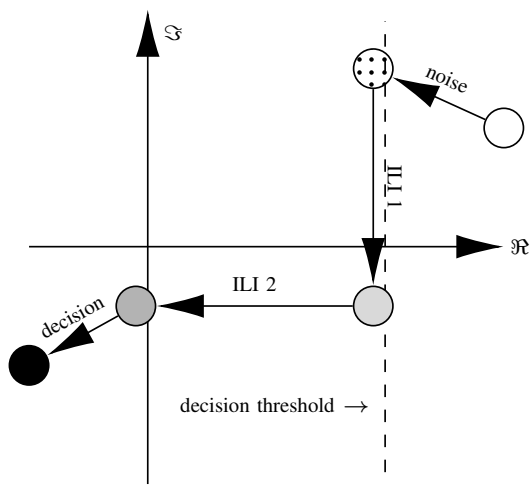

(c) Detecting Layer 3

Fig. 19. Example of detecting subcarriers on layer 1, 2 and 3 of a LACO-OFDM, showing the effect of thermal noise and ILI in the performance. The two axes and the additional dashed line are the decision thresholds for the QAM demapper. White, dotted and black circles represent the transmitted, received and detected symbols, while the gray circles are the intermediate symbols after clipping distortion cancellation of previous layer(s), respectively.

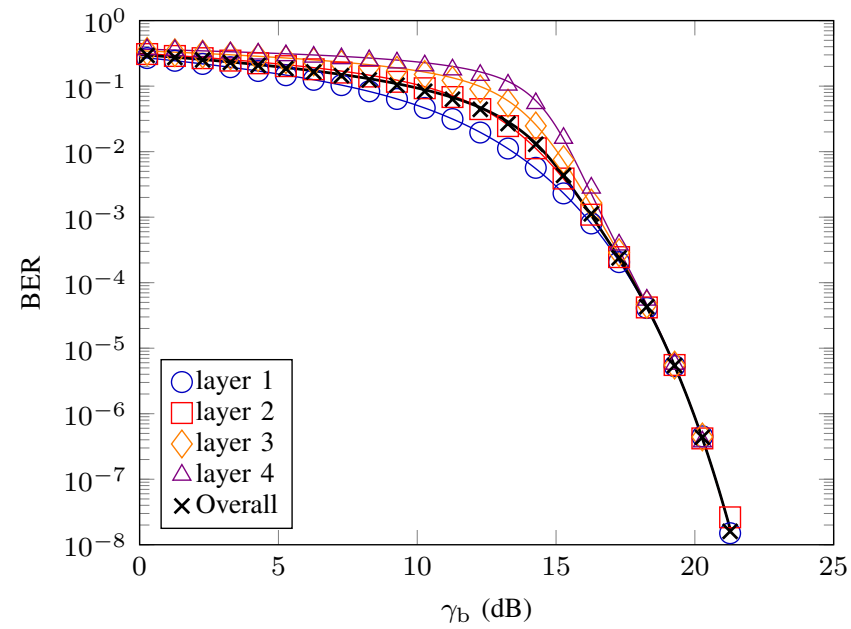

Fig. 20. BER of all layers of 4LACO-OFDM signals for 16QAM modulation. [45]

For layer 1, which is free from ILI, part (e) of (16) can be neglected. Furthermore, if no UB clipping is considered, then part (a) of (16) can be omitted.

It is worth noting that for $l \geq 2$, the $\mathrm{EP} \mathbb{P}_{\mathrm{b}, l}$ of the layer signal always depends on that of its preceding layers $\left(\mathbb{P}_{\mathrm{b}, 1}, \ldots, \mathbb{P}_{\mathrm{b}, l-1}\right)$, due to the cascading ILI term $\sum_{i} \mathcal{G}_{i}$. Therefore, in line with its receiver signal processing, the theoretical BER calculation of LACO-OFDM should also be carried out in a layer-by-layer manner, commencing from layer 1, where $\sum_{i} \mathcal{G}_{i}=0$.

2) BER of Different Layers within the LACO-OFDM Signals: Fig. 20 shows the layer-wise BER vs. $\gamma_{\mathrm{b}}$ performance in 4-layer LACO-OFDM. These curves do not consider the UB clipping of Sec. III-A4. The overall average BER performance of all four layers is also provided. Observe from Fig. 20 that:

- At low SNRs (particularly when $\gamma_{\mathrm{b}}<17 \mathrm{~dB}$ ), different BER performances are exhibited for each layer, with layer 1 having the lowest BER and layer 4 the highest. This is due to the cascaded ILI, which contaminates layer 4 the most drastically, while layer 1 does not suffer from ILI at all.

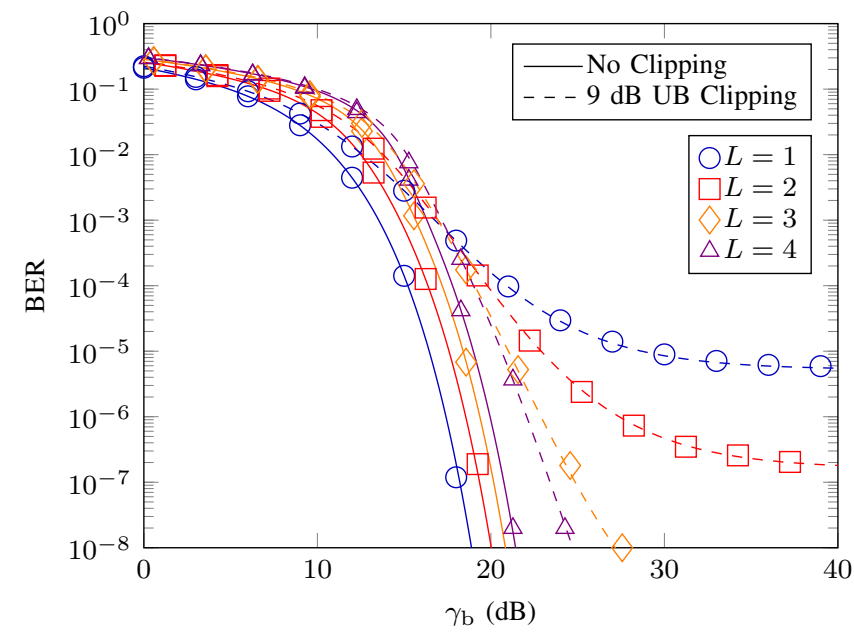

Fig. 21. Overall BER performance of LACO-OFDM signals with different number of total layers $L$ for 16QAM modulation. Dashed lines represent simulations with UB clipping $\tau=9 \mathrm{~dB}$ considered, while solid lines the absence of it. [45]

- At high SNRs (particularly when $\gamma_{\mathrm{b}}>18 \mathrm{~dB}$ ), the four BER curves converge. This is because the improved $\gamma_{b}$ leads to reduced layer BER, which mitigates the ILI regenerated and part (e) in (16) gradually tends to 0 , making $\Gamma_{\mathrm{s}, l}$ for all layers the same.

3) BER of LACO-OFDM for Different Number of Layers: Fig. 21 depicts the overall BER performance of LACOOFDM having different number of layers $L$. The figure shows the performance both with and without UB clipping above $\tau=9 \mathrm{~dB}$. For the cases operating without UB clipping, which are represented by solid lines, degraded BER performance is exhibited by LACO-OFDM composed of more layers, owing to the accumulation of ILI. On the other hand, a reversed trend can observed in cases with the UB clipping, which are shown in dashed lines. The main reason for LACO-OFDM with more layers having lower BER is their lower PAPR, as discussed in Section III-C2, which makes them more robust towards UB clipping. This is more clear as $\Gamma_{\mathrm{s}}$ goes higher, when BER curves of 1 and 2 layers UB-clipped LACO-OFDM 
stops to decrease, since the UB clipping distortion becomes the dominant performance degradation source.

\section{F. Section Summary}

This section covered the receiver design and signal flow of LACO-OFDM, complemented by the theoretical analysis of the BER performance, which was also verified by simulations. The results show that the BER erodes as the number of layers increases, mainly due to the inter-layer interference induced error propagation. In the next section, we will discuss the improvement of the BER performance with the help of forward error correction techniques.

\section{ERror-CORRECTION-AIDED LACO-OFDM}

In Sec. IV-E, the BER performance for LACO-OFDM system has been analyzed. In this section, we focus our attention on improving the performance by employing sophisticated FEC techniques for approaching the channel capacity. While FEC can be simply adopted by applying a single encoder and decoder, the layered structure of LACO-OFDM can be further exploited for improved BER reduction, which will be discussed later in Sections V-C and V-D.

\section{A. Achievable Rate of LACO-OFDM}

In this section, we use the achievable rate to benchmark the BER performance improvement attained by different channel coding schemes. Below we briefly discuss the achievable rate of LACO-OFDM. Since the mathematical derivation is lengthy, we would like to refer motivated readers to the following papers for detailed mathematical insights [49], [53], [68].

For LACO-OFDM, its overall achievable rate can be regarded as the sum of the achievable rates contributed by each of its constituent layers. For the sake of calculating the achievable rate, it is a reasonable approximation to assume an interlayer error-propagation-free system. Then, each layer functions as an independent ACO-OFDM scheme, whose achievable rate would be half that of its immediate preceding layer, due to its halved number of subcarriers. Moreover, the achievable rate of an ACO-OFDM signal would be a quarter of that of an RF-OFDM signal having the same bit-to-symbol mapping and bandwidth, because in ACO-OFDM only a quarter of the subcarriers convey information.

Based on the above discussions, in Fig. 22 we plot the achievable rate of a 4-layer LACO-OFDM scheme relying on different bit-to-symbol mappings.

\section{B. Single-Class Coding}

We firstly conceive a naïve coding system, where the channel coding is simply implemented by cascading the transceiver with the corresponding codec. We refer to this system as a single-class coding scheme, as opposed to the multi-class coding scheme of Sec. V-C.

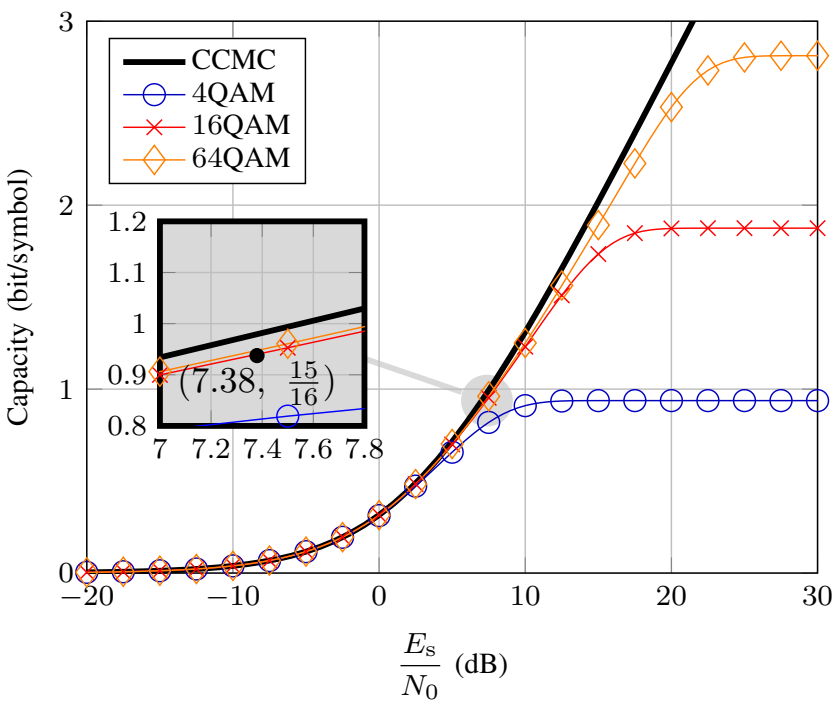

Fig. 22. Achievable rate versus the symbol to noise power ratio of 4-layer LACO-OFDM systems for different QAM constellation sizes [53].

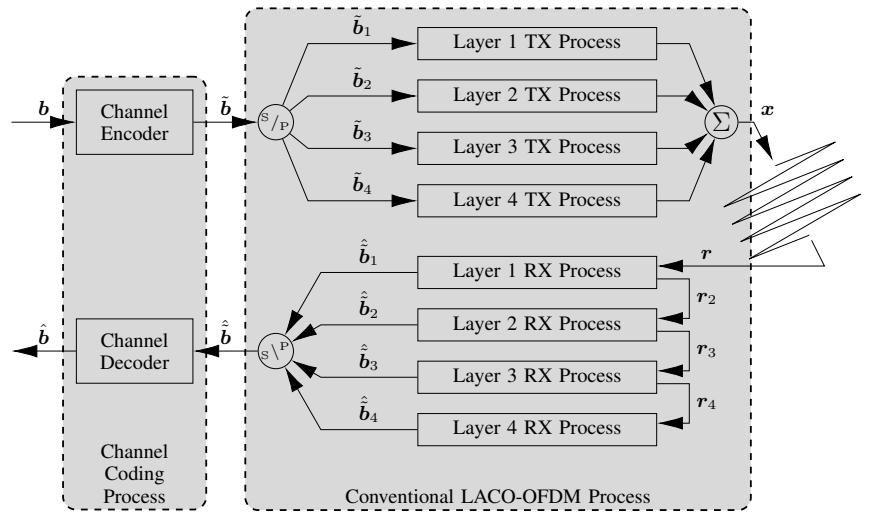

Fig. 23. Single-Class coded LACO-OFDM transceiver for a 4-layer LACOOFDM system.

1) System Model: Fig. 23 depicts the schematic of a single-class LACO-OFDM system. The top half represents the transmitter, where each of the "Layer $l$ TX Process" blocks in the middle uses the ACO-OFDM-style modulation for each layer, similar to those seen in Fig. 10. The bit stream $s$ is passed through the channel encoder, which produces the coded sequence $\tilde{b}$. Then, the LACO-OFDM transmitter separates the single stream into several sub-streams $\tilde{\boldsymbol{b}}_{l}$, each corresponding to a LACO-OFDM layer. The FEC-coded bits are then mapped into symbols and modulated according to Sec. III-A1.

The receiver of the system is seen at the bottom of Fig. 23, where the received signal $\boldsymbol{r}$ is detected on a layer-by-layer manner as in Sec. IV-B, and each of the "Layer $l$ RX Process" stages includes both the layer detection and its clipping distortion cancellation processes, as detailed in Fig. 18. The detected bits of each layer $\hat{\tilde{\boldsymbol{b}}}_{l}$ are combined into a single stream $\hat{\tilde{b}}$ in a chronological order before channel decoding, resulting in $\hat{b}$ of Fig. 23. Again, as shown in Fig. 23, in this singleclass scheme, the channel coding components receive the harddecision bit stream from the LACO-OFDM scheme. 


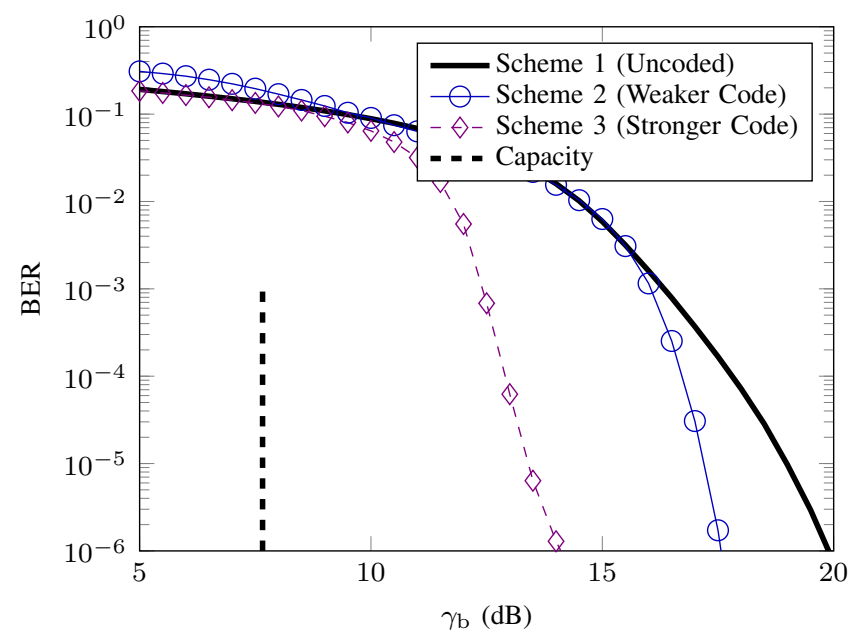

Fig. 24. BER performance of 4-layer single-class coded LACO-OFDM for 16QAM modulation. Details about the simulation schemes are also summarized in Tab. VIII.

Next, we will investigate the error correction capability of this single-class coded scheme by carrying out Monte-Carlo simulations for various FEC codes.

2) Numerical Results: The error correction capability of single-class coding is evaluated in this section. We have used a 4-layer LACO-OFDM scheme communicating through an AWGN channel relying on a total of 2048 subcarriers and 16QAM on all 4 layers. Regardless of the choice of FEC code, the coding rate is always $\mathrm{R}=\frac{1}{2}$. The corresponding channel capacity is $\gamma_{\mathrm{b}}=7.66 \mathrm{~dB}$, as detailed in [45].

Fig. 24 shows the BER performance of a LACO-OFDM system employing the above-mentioned single-class scheme, where the choice of FEC codes is varied. A weaker convolutional code with memory $\mathcal{M}=2$ (Scheme 2 ) and the octally represented generator polynomial $(7,5)$ as well as a code-rate of $\mathrm{R}=\frac{1}{2}$ is used. A stronger turbo code relying on a pair of $\mathcal{M}=3$ Recursive Systematic Convolutional (RSC) codes (Scheme 3) using the generator polynomials $(15,13)$ and a maximum of $\mathcal{I}=8$ decoding iterations is also employed for comparison. The coding rate was again $\mathrm{R}=\frac{1}{2}$ after puncturing. The performance is also compared against the uncoded results of Scheme 1 characterized in Fig. 21, as well as to the $7.66 \mathrm{~dB}$ capacity threshold.

As shown in Fig. 24, utilizing the single-class coding system does improve the BER performance of our LACO-OFDM system. Explicitly, employing the $\mathcal{M}=3$ convolutional code (Scheme 2) starts to produce a lower BER than its uncoded counterpart (Scheme 1) for SNR beyond $\gamma_{\mathrm{b}} \approx 15.5 \mathrm{~dB}$, while having a coding gain of around $0.25 \mathrm{~dB}$ at $\mathbb{P}_{\mathrm{b}}=10^{-3}$. Moreover, the performance can be further improved by the $\mathcal{I}=8, \mathcal{M}=2$ turbo code (Scheme 3), which outperforms its uncoded counterpart for SNRs in exceeds of $\gamma_{\mathrm{b}}=8.5 \mathrm{~dB}$, giving a $3.9 \mathrm{~dB}$ gain at $\mathbb{P}_{\mathrm{b}}=10^{-3}$.

3) Discussions: Although the single-class coded LACOOFDM exhibits a slightly improved BER performance, it also has some impediments:

(a) Lack of Parallelization: In the design of the original
LACO-OFDM TX of Figs. 9 and 10, the processings of each layer is carried out in parallel. In the single-class coded LACO-OFDM, the encoder precedes the parallel "ACO TX" blocks. The higher layers' processors then have to wait until their bits have been encoded, which introduces undesired delay processing.

(b) Lack of Flexibility: A single encoder has the same coding rate for all layers. Therefore, it is impossible to provide higher protection for a subgroup of layers by invoking stronger codes. Even though the flexibility of LACO-OFDM is beneficial, using a single FEC code reduces its flexibility.

(c) Inability to Mitigate ILI: The single-class FEC scheme also fails to mitigate the ILI, because there is only a single decoder at the RX, which is placed after the P/S block of Fig. 23. In order to mitigate the error propagation, the error correction must be carried out before the clipping distortion cancellation stage of Fig. 18.

Therefore, more sophisticated FEC designs would be required to address these issue, such as for example, the multi-class coded and the multi-layered coded LACO-OFDM schemes introduced below.

\section{Multi-Class Coding}

In order to further exploit the potential of FEC and mitigate the ILI, we introduce the multi-class coded LACO-OFDM architecture of Figs. 25 and 26 in this section. Explicitly, multiple FEC components are employed, namely a dedicated one for each layer of LACO-OFDM, as opposed to the singleclass architecture of Fig. 23.

1) Transmitter Design: Fig. 25 shows the transmitter block diagram of multi-class coded LACO-OFDM. After splitting the original bit stream $\boldsymbol{b}$ in to $L$ sub-streams (the example in Fig. 25 uses $L=4$ ), each layer employs its own FEC encoder to produce the encoded stream $\tilde{\boldsymbol{b}}_{l}$, which may have different coding rates. The coded streams are then forwarded to the "TX Process" blocks for further ACO-OFDM TX processing, which is the same as in the original LACO-OFDM TX of either Fig. 9 or Fig. 10.

In contrast to the single-class coded LACO-OFDM TX of Fig. 23, the multi-class coded TX is now capable of simultaneously processing both the encoding and the modulation of each layer. As a further benefit, it is now possible for each layer to have different FEC coding rates, thus further improving the flexibility of LACO-OFDM.

2) Receiver Design: The receiver design of the multi-class coded LACO-OFDM is shown in Fig. 26, featuring the same iterative detection philosophy as in the original receiver of Fig. 18. Additionally, the detection-regeneration-cancellation loop of each layer of the multi-class coded receiver of Fig. 26 also contains an FEC decoder, which is capable of correcting the detection errors imposed by the "ACO RX" blocks before regenerating the clipping distortion at "ACO TX". Furthermore, instead of providing the hard bit estimates $\hat{\boldsymbol{b}}_{l}$, the "ACO RX" block of each layer calculates the a priori soft information of the bit stream $\mathcal{L}_{\mathrm{a}}\left(\hat{\boldsymbol{b}}_{l}\right)$ for the FEC decoder. 


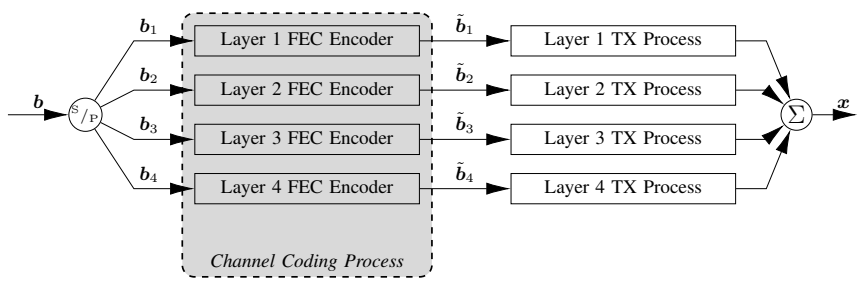

Fig. 25. A LACO-OFDM transmitter integrated with FEC encoders, where the "TX Process" blocks can be either Fig. 9 or Fig. 10.

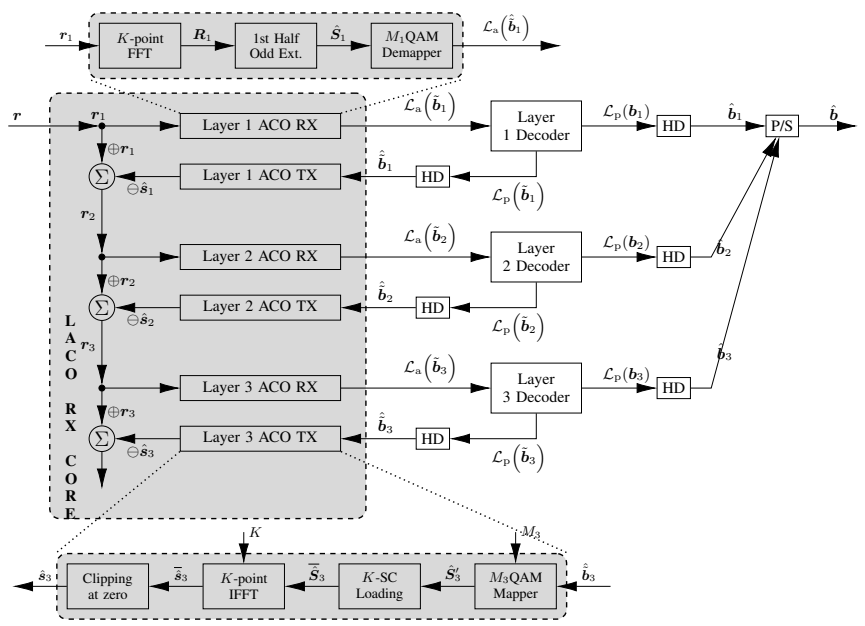

Fig. 26. Multi-Class Coded LACO-OFDM RX, where $\mathcal{L}_{\mathrm{a}}(\cdot)$ and $\mathcal{L}_{\mathrm{p}}(\cdot)$ are the a prior and a posteriori soft information of the stream $(\cdot)$, while "HD" stands for hard-decision converting soft information into hard bits.

The FEC decoder of each layer seen in Fig. 26 carries out error correction and produces a posteriori soft information for a pair of bit streams: namely for $\mathcal{L}_{\mathrm{p}}\left(\boldsymbol{b}_{l}\right)$ of the uncoded stream and for $\mathcal{L}_{\mathrm{p}}\left(\tilde{\boldsymbol{b}}_{l}\right)$ of the coded stream, both of which are then converted into their hard-decision counterparts $\hat{\tilde{\boldsymbol{b}}}_{l}$ and $\hat{\boldsymbol{b}}_{l}$. The error-free stream $\hat{\tilde{\boldsymbol{b}}}_{l}$ is then forwarded to the "Layer $l$ ACO TX" block of Fig. 26 for regenerating the clipping distortion to be canceled out from $\boldsymbol{r}_{l}$ and for producing $\boldsymbol{r}_{l+1}$, for the detection-regeneration-cancellation loop of the next layer.

The FEC decoder of each layer corrects most of the thermalnoise-imposed errors, hence preventing inter-layer error propagation and reducing the ILI before the regeneration process. Therefore, the multi-class coded design of Figs. 25 and 26 is expected to outperform the single-class coded one of Fig. 23.

3) Numerical Results and Discussion: In this section, we characterize the performance of the multi-class coded LACOOFDM and compare it against its single-class counterpart using the same simulation parameters as in Section V-B2.

More explicitly, in Fig. 27, we compare the error ability of a weaker and a stronger FEC code, as detailed in Tab. VIII. The performance is also benchmarked against the $7.66 \mathrm{~dB}$ capacity bound. The complexity of the single-class scheme is simply the number of trellis-states multiplied by the number of bits per LACO-OFDM symbol. By contrast, this principle has to be applied to each class separately, if they use different FEC codes. Moreover, if we have the same FEC codec for each layer, the multi-class scheme has identical complexity to that of the single-class scheme, because the total number of bits they are handling remains unchanged. It is clear from Fig. 27 that the multi-class scheme outperforms the singleclass scheme for both the weaker convolutional code and for the stronger turbo code, thanks to its ability to mitigate error propagation at RX. More importantly, by comparing Schemes 3 and 4, it becomes clear that even a simple 4-state convolutional code of the multi-class scheme has a better BER performance than a sophisticated 128-state turbo code singleclass coding scheme, which has 32 times higher complexity.

To expound a little further, it is also possible to study the performance of each individual layer as seen in Fig. 28, where Scheme 5 of Tab. VIII was adopted. The results are contrasted to the uncoded BER performance of Fig. 20, which shows a drastic BER reduction for all layers. Moreover, the four BER curves no long converge at high SNRs. Explicitly, even when the same bit-to-symbol mapping scheme, the same FEC code and the same coding rate are used, layer 1 has the lowest BER, because it contains the highest number of bits, and therefore the FEC code can invoke a longer interleaver, which in turn improves the BER performance.

By contrast, the upper layers have a higher BER owing to their shorter interleaver. This can be exploited for example for unequal protection of audio [80] and video [81], [82] signals. However, in conventional data-streaming, all layers should be offered the same level of protection. Therefore, in the next section we present a design with this in mind. 
TABLE VIII

Channel Coding Schemes Used for Simulations

\begin{tabular}{|c|c|c|c|c|c|c|}
\hline Scheme & Structure & Code & Parameter & Rate & Polynomials & States \\
\hline 1 & \multicolumn{6}{|c|}{ No FEC } \\
\hline 2 & \multirow{2}{*}{ Single-Class } & Convolutional & $\mathcal{M}=2$ & \multirow{4}{*}{$\frac{1}{2}$} & $(7,5)$ & 4 \\
\hline 3 & & Turbo & $\mathcal{M}=3, \mathcal{I}=8$ & & $(15,13)$ & 128 \\
\hline 4 & \multirow{2}{*}{ Multi-Class } & Convolutional & $\mathcal{M}=2$ & & $(7,5)$ & 4 \\
\hline 5 & & Turbo & $\mathcal{M}=3, \mathcal{I}=8$ & & $(15,13)$ & 128 \\
\hline
\end{tabular}

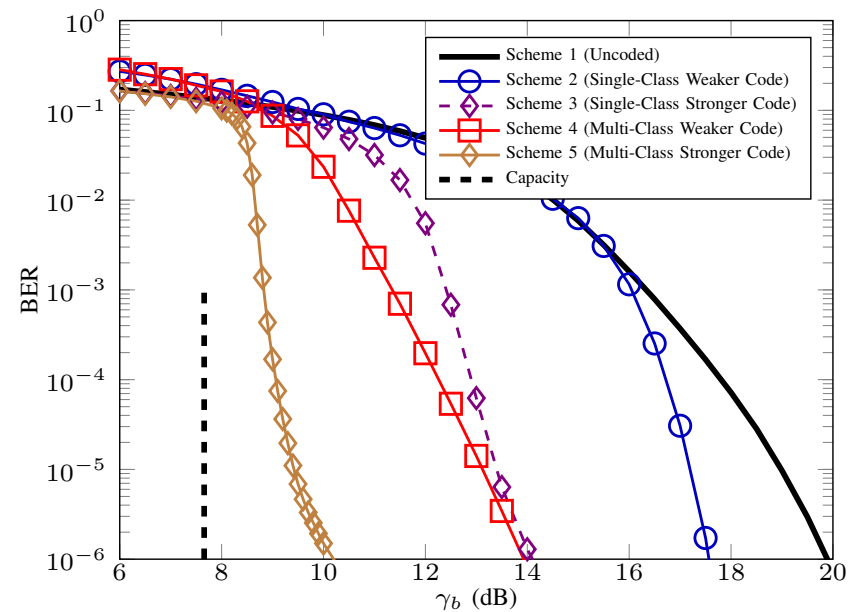

Fig. 27. BER performance of a 4-layer LACO-OFDM system operating 16QAM on all of its layers communicating in AWGN channels. The channel coding schemes of Tab. VIII are used.

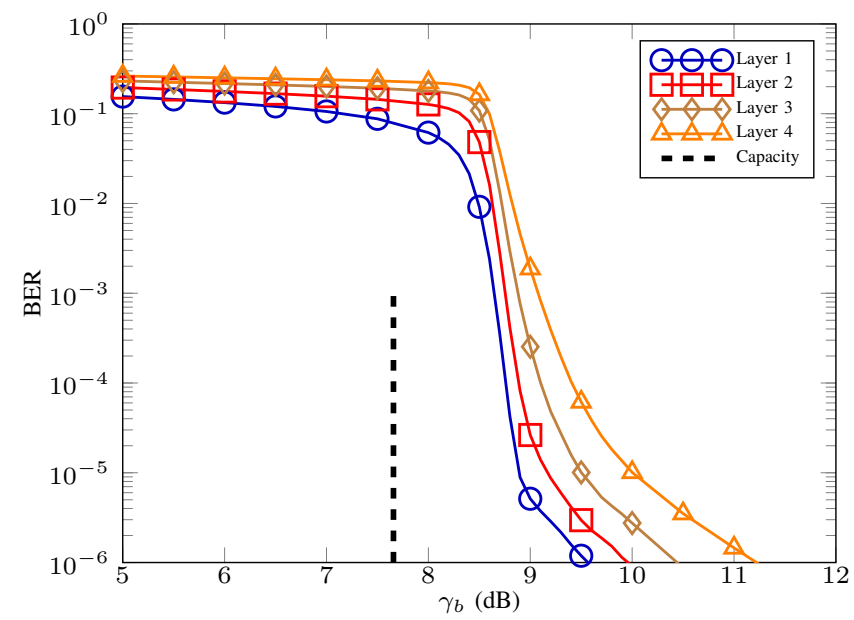

Fig. 28. BER performance on each layer of a 4-layer LACO-OFDM system operating 16QAM on all of its layers communicating in AWGN channels with 8-iteration turbo code, and each layer having its maximum achievable interleaver length. [53]

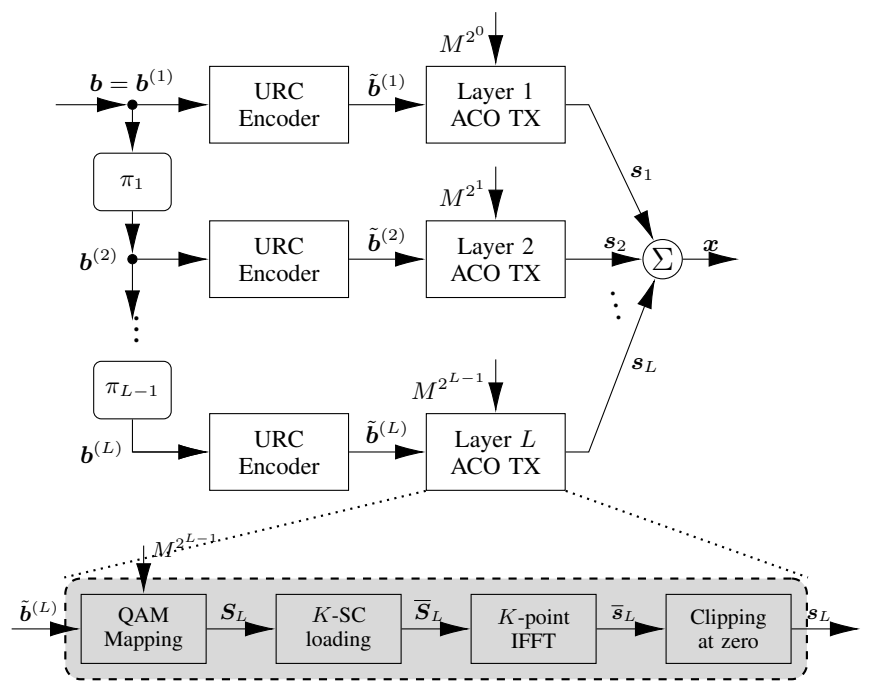

Fig. 29. Schematic of multi-layered coded LACO-OFDM transmitter, where $\pi_{l}$ is the $l$ th interleaver.

\section{Multi-Layered Coding}

In contrast to multi-class coding, the multi-layered coding philosophy provides the same protection for all bits without perturbing the layered structure of LACO-OFDM. The main objective of harvesting the upper layers is to incorporate more parity information for increasing the robustness against transmission errors, rather than increasing the effective information throughput. More explicitly, the multi-class coding confines both the information and the redundancy to individual layers, but dispenses with any inter-layer processing. By contrast, the multi-layered coding scheme exploits the potential of the layered structure, while ensuring the same protection for all bits, as detailed below.

1) Transmitter Design: In the multi-layered coded LACOOFDM, Unity Rate Codes (URC) [83], [84] are adopted as the FEC component for each layer. As the terminology suggests, an URC scheme has a coding rate of 1, thus does not change the length of the bit stream after encoding. Despite its unity rate, it is capable of providing error protection for the information.

Fig. 29 shows the schematic of the multi-layered coded LACO-OFDM transmitter, where $L$ differently interleaved copies of the information stream $\boldsymbol{b}$, namely $\left\{\boldsymbol{b}^{(1)}, \boldsymbol{b}^{(2)}, \ldots, \boldsymbol{b}^{(L)}\right\}$, are transmitted over the $L$ layers, respectively. On layer $l$, the URC encoder produces the coded stream $\tilde{\boldsymbol{b}}^{(l)}$, which is forwarded to the "Layer $l$ ACO TX" for 
the same processing as in the multi-class coding transmitter of Fig. 25. However, since each layer has been assigned only half the number of subcarriers compared to the previous one, the number of bits per symbol must be doubled. For example, if layer 1 used Binary Phase Shift Keying (BPSK), layer 2 has to use Quadrature PSK (QPSK), etc. To generalize, if layer 1 adopted $M$-ary QAM, layer $l$ has to employ an $M^{2^{l-1}}$-ary QAM for accommodating the same number of bits.

2) Receiver Design: Fig. 30 shows the multi-layered coded LACO-OFDM receiver. Explicitly, the detection-regenerationcancellation loops of each layer within the gray-shaded "LACO RX CORE" area are exactly the same as the multiclass coded receiver of Fig. 26. However, the multi-layered coded receiver handles FEC decoding differently. Although the bit-to-symbol and bit-to-layer mapping is different for each layer, the same information is passed through all layers. The advantage of this sophisticated scheme is that the decoding of the current layer can benefit from the additional information provided by the other layers' detection. Explicitly, the decoder of Fig. 30 takes two inputs:

- the soft information of the coded bit stream $\mathcal{L}_{\mathrm{a}}\left(\tilde{\boldsymbol{b}}^{(l)}\right)$ like in multi-class coding, and

- the soft information of the uncoded bit stream $\mathcal{L}_{\mathrm{a}}\left(\boldsymbol{b}^{(l)}\right)$ obtained from the decoding results of all other $(L-1)$ layers.

When activating the RX process of Fig. 30 for the first time, the decoder of each layer generates the soft extrinsic information $\mathcal{L}_{\mathrm{e}}\left(\boldsymbol{b}^{(l)}\right)$ with the aid of its FEC components. As the process moves on to the next layer, $\mathcal{L}_{\mathrm{e}}\left(\boldsymbol{b}^{(l)}\right)$ is fed into the decoder of layer $(l+1)$ as the soft information representing the uncoded bit stream $\mathcal{L}_{\mathrm{a}}\left(\boldsymbol{b}^{(l)}\right)$ after the interleaving process denoted by $\pi_{l}$ of Fig. 30 as it did at the TX side. Once all layers have been detected, the extrinsic information provided by the higher layers can then be fed back for improving the detection results of the lower layers.

For this purpose, a second iteration of the RX process is carried out. This time, the uncoded soft information $\mathcal{L}_{\mathrm{a}}\left(\boldsymbol{b}^{(l)}\right)$ entered into the decoder will be composed of the extrinsic soft information $\mathcal{L}_{\mathrm{e}}\left(\boldsymbol{b}^{(l-1)}\right)$ gleaned both from the previous layer and from $\mathcal{L}_{\mathrm{e}}\left(\boldsymbol{b}^{(l+1)}\right)$ of the next layer, where the former is obtained in the current iteration, while the latter is carried forward from the previous iteration. This process of extrinsic information exchange can be applied for several iterations, and each round yields an additional performance improvement in the spirit of [85]. Finally, after sufficiently high number of iterations, the final output $\mathcal{L}_{\mathrm{p}}(\boldsymbol{b})$ can be obtained by combining the extrinsic information $\mathcal{L}_{\mathrm{e}}\left(\boldsymbol{b}^{(1)}\right)$ of layer 1 and the a priori information $\mathcal{L}_{\mathrm{a}}\left(\boldsymbol{b}^{(1)}\right)$.

3) Numerical Results: The BER performance of multilayered coded LACO-OFDM is evaluated in Fig. 31 and marked by circles. In these simulations, we used 3-layer LACO-OFDM relying on 2024-point IFFT/FFT, while the FEC components are the binary memory-1 URCs relying on the octally represented feedback polynomial $(2,3)_{\text {ocl }}$. Then, 2QAM is adopted for layer 1 , which implies that 4QAM and 16QAM are employed for layers 2 and 3, respectively, yielding an equivalent $\mathrm{BE}$ of $0.25 \mathrm{bit} / \mathrm{s} / \mathrm{Hz}$. The channel capacity [45] is also shown in Fig. 31 as a benchmark. It can be observed from Fig. 31 that as the number of iterations increases from 1 to 10 , the corresponding BER performance gradually improves, with 10-iteration multi-layered coding having only $2 \mathrm{~dB}$ distance from the channel capacity at $\mathbb{P}_{\mathrm{b}}=10^{-4}$.

The performance of the multi-class and multi-layered coding schemes is also compared in Fig. 31, where multi-class coding uses 3-layer LACO-OFDM relying on BPSK modulation and half-rate URCs codes on each layer, yielding a slightly lower equivalent throughput of $0.21875 \mathrm{bit} / \mathrm{s} / \mathrm{Hz}$. For a fair comparison, multiple URCs are cascaded in our multi-class coding scheme to align the complexity with that of the multi-iteration, multi-layered coding scheme. Observe in Fig. 31 that, multi-layered coding outperforms its multiclass counterpart at the same complexity. It is also worth emphasizing again that multi-layered coding provides equal protection for all bits, which is not the case for multi-class coding.

\section{E. Performance over Frequency Selective Optical Fiber Chan- nel}

In this section we briefly highlight a LACO-OFDM transmission experiment over optical fiber based on [69], where 3-layer LACO-OFDM waveform was amplified before it was forwarded to a Mach-Zehnder intensity Modulator (MZM) for electrical-to-optical conversion. This signal was then transmitted by a continuous-wave laser over a single mode fiber having a lnegth of $10 \mathrm{~km}$ and it was detected by a photodiode, before it was amplified in the electronic domain, followed by analogue-to-digital conversion and LACO-OFDM detection. Give the non-flat frequency-domain transfer function of the fiber, frequency-dependent adaptive bit-loading was used. The results indicate the feasibility of practical high-speed fiber based transmission of LACO-OFDM signals, where a significant coding gain of about $4.22 \mathrm{~dB}$ was attained at a BER of $4.7 \times 10^{-3}$ for the multi-class coding scheme of [69].

\section{F. Section Summary}

In this section, we covered three bespoke channel coding designs conceived for LACO-OFDM, namely the single-class, multi-class and multi-layered coding systems. Their performances were also verified both by simulations and experiments, while their pros and cons were presented in Tab. IX. A range of other performance improvement techniques will be considered in the next section.

\section{Additional PERformance IMPROVEMENT TECHNIQUES}

In this section, we briefly introduce some notable breakthroughs in the context of LACO-OFDM since its conception on [29], noting that its history has been provided in Tab. III.

\section{A. Lower-Bound Clipping Distortion and Inter-Layer Interfer- ence}

Wang et al. [41] conceived an improved LACO-OFDM receiver by further exploring the characteristics of the TD 


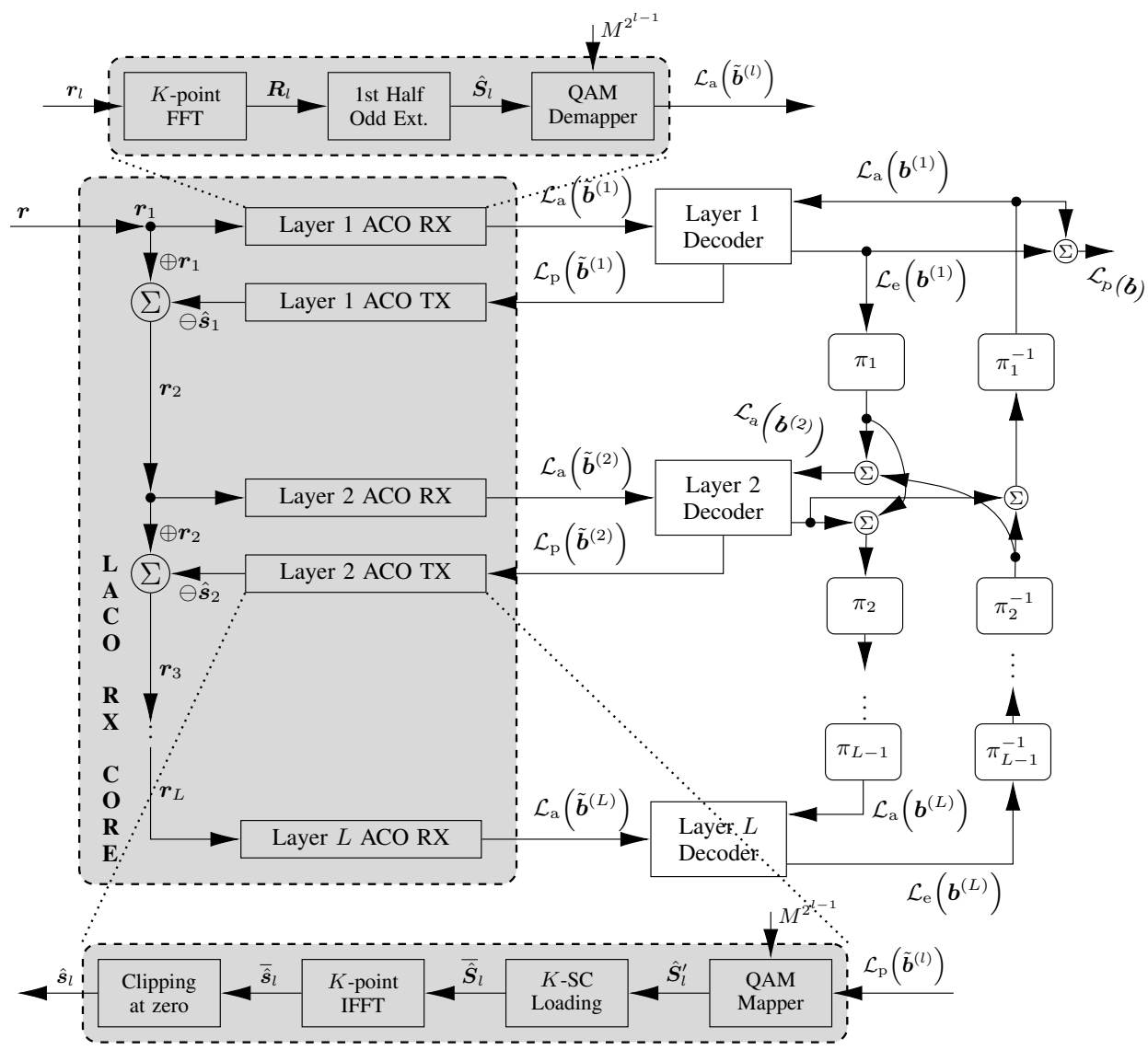

Fig. 30. Schematic of multi-layered coded LACO-OFDM receiver, where each layer decoder takes a priori soft information of both the coded bit stream $\mathcal{L}_{\mathrm{a}}\left(\tilde{\boldsymbol{b}}^{(l)}\right)$ as well as uncoded bit stream $\mathcal{L}_{\mathrm{a}}\left(\boldsymbol{b}^{(l)}\right)$, and produces the soft a posteriori information of the coded bit stream $\mathcal{L}_{\mathrm{p}}\left(\tilde{\boldsymbol{b}}^{(l)}\right)$ as well as the extrinsic information of the uncoded bit stream $\mathcal{L}_{\mathrm{e}}\left(\boldsymbol{b}^{(l)}\right)$, while $\pi^{-1}$ are the reverse interleavers corresponding to those found in Fig. 29.

TABLE IX

Comparison of Single-Class, Multi-Class and Multi-Layered Coding Systems

\begin{tabular}{llll}
\hline Aspect & Single-Class & Multi-Class & Multi-Layered \\
\hline Design & $\begin{array}{l}\text { easy } \\
\text { simple encoder and de- } \\
\text { coder concatenation }\end{array}$ & $\begin{array}{l}\text { moderate } \\
\text { adding encoders and de- } \\
\text { coders on all layers }\end{array}$ & $\begin{array}{l}\text { hard } \\
\text { complete redesign of the } \\
\text { system structure }\end{array}$ \\
\hline Computational Complexity & same & same & same \\
\hline Time Delay & $\begin{array}{l}\text { long } \\
\text { serial encoding }\end{array}$ & $\begin{array}{l}\text { short } \\
\text { parallel encoding }\end{array}$ & $\begin{array}{l}\text { shorter } \\
\text { parallel encoding and iter- } \\
\text { ative decoding }\end{array}$ \\
\hline BER Performance & bad, Fig. 24 & good, Fig. 27 & better, Fig. 31 \\
\hline
\end{tabular}

LACO-OFDM signals. The original receiver of [29] detects the signal from layer 1 upwards to layer $L$, successively canceling out the distortion generated by each layer after detecting and re-modulating it. By contrast, the receiver proposed in [41] conducts an additional round of interference cancellation for improving the attainable BER at the cost of an increased complexity.

When canceling out the clipping distortion imposed on each layer, the original receiver of [29] relied on hard decisions. In doing so, the receiver remaps the detected bits of each layer back to QAM symbols for reproducing the clipping distortion. By contrast, in [42], Wang et al. used soft decision based Successive Interference Cancellation (SIC) at the receiver. The soft-value of each bit is used for reproducing the clipping distortion, rather than the bits after hard-decisions. Simulations were performed for characterizing the scheme advocated, demonstrating that this scheme mitigated the error propagation imposed by hard-decisions. This scheme was even further improved by Wang et al. [42], resulting in reduced residual interference and an improved BER. As a further development, Mohammed et al. [43] proposed to use diversity combining for improving the performance. Simulations were conducted for transmission over both flat and frequency selective channels to verify the improved BER performance. 


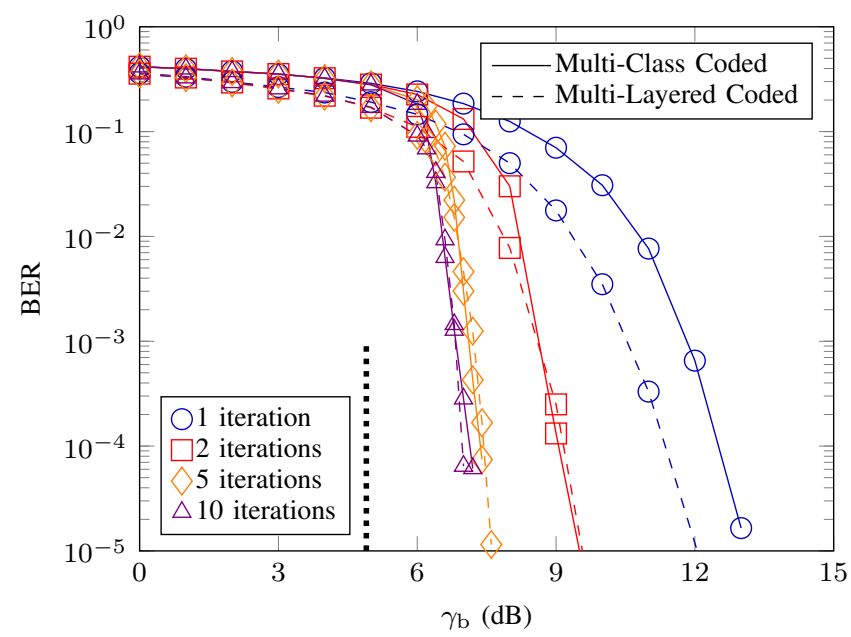

Fig. 31. Achievable BER performance of 3-layer multi-layered coded LACOOFDM system of Figs. 29 and 30, having $M=2$ and binary memory-1 URCs. We have used $K=2048$ subcarriers and interleavers of length 4096 (equivalent to eight LACO-OFDM frames). [58]

\section{B. Upper-Bound Clipping Distortion and Peak-to-Average Power Ratio}

Sun et al. [44] analyzed the PDF, PAPR as well as the BER performance of LACO-OFDM, and compared its performance to both ADO-OFDM [24] and Hybrid ACO-OFDM (HACOOFDM) [25].

In [48], Zhou et al. designed a layered scheme similar to LACO-OFDM. Instead of using QAM based ACO-OFDM on each layer as in the original LACO-OFDM [29], the scheme of [48] applied discrete Hartley transform (DHT)-based OFDM for PAM symbols on each layer, resulting in Single-Carrier Frequency Domain Multiplexed (SCFDM) layered signals. These signals were then clipped and superimposed in the TX. At the receiver, they were detected in the same way as in the original LACO-OFDM of [29]. Finally, its performance analysis was carried out for demonstrating its reduced computational complexity and PAPR, while the simulations provided revealed its higher immunity to both transmitter nonlinearity and to the multi-path fading.

In [52], Wang et al. studied the UB clipping distortion in LACO-OFDM by modeling the distortions imposed on each layer using Bussgang's theorem, which led to an accurate mathematical expression of the distortion. Using the results derived, an improved LACO-OFDM receiver was proposed, which is capable of identifying the peaks that are UB-clipped and therefore it makes improved decisions. Their simulations demonstrated improved BER performance under clipping.

\section{Complexity Reduction}

Since each layer of LACO-OFDM is constituted by an ACO-OFDM scheme, half of whose FD subcarrier is empty and it is rather wasteful to apply full-length IFFT at the transmitter. In [86], Wang et al. carefully analyzed this process and improved the transmitter design of LACO-OFDM by simplifying the IFFT stage and removing the branches handling the blank subcarriers.
In [57], Li et al. improved the transmitter signal processing of the LACO-OFDM signal. Explicitly, in their TX design, the higher layer signals were pre-distorted by subtracting the clipping distortions of the previous layers from the signal before LB clipping. Therefore, the signals became free from clipping distortion contamination at the receiver side and all layers were directly detected. This new scheme was referred to as Hierarchical Pre-Distorted LACO-OFDM (HPD-LACOOFDM). Their work solution prevented the error propagation from the transmitter side and resulted in outstanding BER performance, despite its complexity reduction at the RX.

\section{Higher System Capacity}

Recently, there have been substantial research efforts dedicated to further improving the O-OFDM system capacity by harvesting the Non-Orthogonal Multiple Access (NOMA) concept [87]. A NOMA-O-OFDM system allows multiple users to access the network replying on the same time- and frequencyresource slots, and separating them on the basis of their unique power-difference. Hence, this technique is termed as power domain multiplexing. At the receiver, the higher-power usersignal is detected first by simply considering the lower-power signal as interference. The resultant higher-power signal is then remodulated and then subtracted from the composite signal, leaving the clean uncontaminated signal behind. This technique beneficially increases the overall network capacity [88]. However, the inter-cell interference (ICI) imposed by densely deploying the user terminals and access points also has be mitigated by carefully adapting the techniques originally conceived for RF-OFDM. In [56], a multi-user NOMA-LACOOFDM scheme was proposed by Feng et al., where the signal of the users superimposed at different powers was separated in the power domain. In this treatise, a hybrid OMA-NOMA scheme was employed, where the classic timedivision multiple access (TDMA) based scheduling was used for combating the ICI. At the receiver, the detector employed a pair of nested loops, where the inner loop processed the signal of the different users as in conventional RF-NOMA, while the outer loop mitigated the clipping distortion and ILI, as in single-user LACO-OFDM. The problems of power sharing and user-scheduling were also solved and the simulation results verified the accuracy of the theory. In [57], Li et al. applied the NOMA concept to their proposed hierarchical pre-distorted LACO-OFDM in the same manner as in [56], but succeeded in mitigating the source of ILI, which is a benefit brought about by the transmitter-side pre-distortion invoked at the transmitter. Hence, they improved the BER performance in their conventional NOMA LACO-OFDM benchmark system.

\section{Conclusions And Design Guidelines}

In this section, we provide a bird's-eye perspective of the trade-offs in designing a LACO-OFDM communication system, and summarize the main points of this paper.

\section{A. Design Trade-Offs}

As discussed in Sec. II-C, the inspiration of LACO-OFDM is to strike a trade-off between the power and bandwidth 
Available Techniques

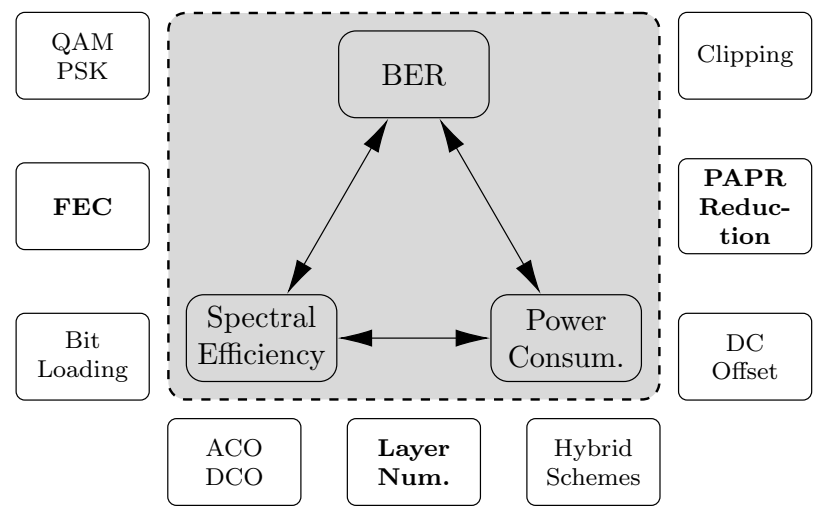

Fig. 32. Relationship of the techniques mentioned in this paper for LACOOFDM, resulting in different design trade-offs.

efficiencies of ACO-OFDM and DCO-OFDM. While ACOOFDM avoids the requirement of a substantial DC offset and hence achieves power-efficient transmission, this is at the cost of reducing the $\mathrm{BE}$ by a factor of two, again, striking a trade-off between the power and bandwidth efficiency. The relationship of various techniques discussed throughout this treatise are summarized in Fig. 32.

The rest of this subsection discusses the three trade-off pairs depicted in Fig. 32 with reference to the topics mentioned in the previous sections.

- The amount of information that may be conveyed at a given $\mathrm{PE}$ is directly related to the $\mathrm{BE}$.

- Recall from Fig. 5 that ACO-OFDM and DCO-OFDM may be deemed to have a high $\mathrm{BE}$ and $\mathrm{PE}$, respectively. Therefore, the simplest way to improve the $\mathrm{PE}$ is to switch from DCO-OFDM to using ACO-OFDM, while reconfiguration the other way around improves the $\mathrm{BE}$.

- Hybrid O-OFDM schemes, such as ADO-OFDM [24], HACO-OFDM [25] etc., strike a compromise in terms of the BE and PE, compared to ACO-OFDM and DCOOFDM, because they are designed to amalgamate the advantages of the basic O-OFDM schemes by relying on the hybrid contribution of a pair of schemes.

- As one of the most compelling solutions in terms of striking an attractive BE-PE trade-off, LACO-OFDM provides even higher flexibility, since the number of layers $L$ can be readily adapted in response to different operational requirements. In the example of Fig. 5, the dotted lines represent LACO-OFDM schemes having different number of layers $L$. By increasing $L$, the $\mathrm{BE}$ curves gradually move from the low-BE-efficiency ACO-OFDM scheme towards the high-BE-efficiency DCO-OFDM arrangement. By contrast, the PE curves would move in the opposite direction, as we increase the $\mathrm{BE}$ by using more layers at the cost of more power.

- Naturally, in LACO-OFDM, the relationship between the BER and PE also has to obey a trade-off, where generally a reduced BER can be achieved at increased power consumption.

- The relationship between the BER performance and the channel conditions (i.e. SNR, SINR) have been characterized in (14) and Fig. 20.

- On the other hand, due to the limitation of the linear range in optical front-end components (such as the LEDs), the signal at high power inevitably suffers from non-linear distortions. Hence, UB clipping must be used, but unfortunately this increases the BER.

- A problem closely related to the UB clipping is the PAPR of the signal, where the UB clipping distortion can be reduced when the signal has a lower PAPR, leading to a better BER performance. Therefore, PAPR reduction methods, such as tone-injection [45] or more advanced power sharing [89] can be used.

- Finally, we discuss the trade-off between the BE and BER.

- The most obvious relationship between the BE and BER is related to the specific choice of the QAM constellation utilized for each subcarrier. According to Section IV-E1, given the total power and the number of layers $L$, by reducing the number of information bits conveyed, upon reducing the modulation order and the $\mathrm{BE}$, the Hamming distance between the constellation points is increased, which in turn reduces the BER.

- Another popular performance improvement technique is constituted by FEC. As covered in Section V, a powerful FEC is capable of drastically reducing the BER, provided that the SNR is high enough. However, the transmitter has to send additional redundant bits for error correction, which reduces the $\mathrm{BE}$, since the portion of information bits is reduced and so is the energy per bit.

\section{B. Conclusions and Design Guidelines}

We have provided a historic perspective on the evolution of optical OFDM from its roots to its most flexible incarnation, namely LACO-OFDM. Both the transmitter and receiver architectures have been described and the signal processing flow between them has been visualized with the aid of both TD and FD graphical examples. The LACO-OFDM signals have been characterized by their PDF and PAPR. Furthermore, its BER performance has been characterized. Then, the family of suitable FEC schemes has been surveyed and their performances have been compared at the same overall complexity. A range of other optical OFDM improvements have also been surveyed, highlighting some of the open problems requiring future research.

To construct an optical communications system using LACO-OFDM, the following design guidelines have to be considered. Firstly, it is of vital importance to determine the specific choice of metric with the highest priority for optimization. Specifically, in the trade-off between BE and PE, if $\mathrm{BE}$ is to be prioritized, LACO-OFDM having a high number of layers is recommended, bearing in mind the maximum affordable complexity and power. High-order modulation may be used for each layer to further boost the BE, albeit - naturally 


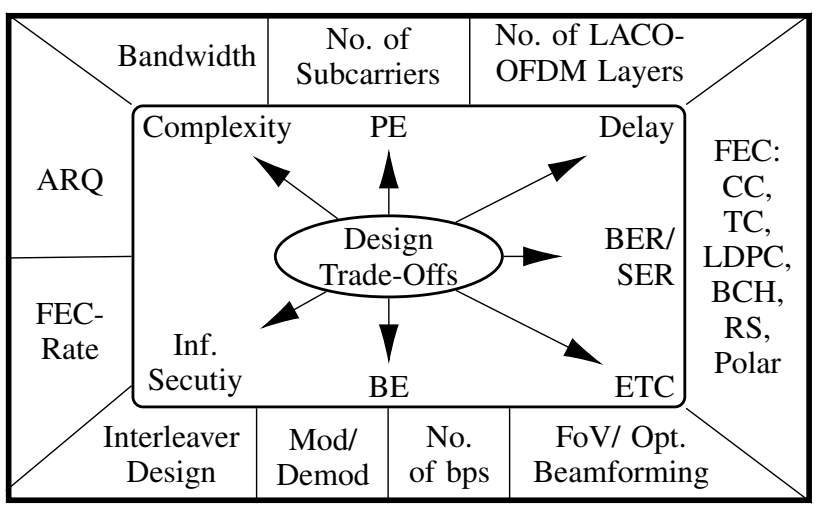

Fig. 33. Design guidelines for LACO-OFDM.

- at a cost of reducing the PE. By contrast, should the PE be the main consideration, a low number of layers tending to a single one should be used, when LACO-OFDM degenerates to ACO-OFDM.

To elaborate a little further on the associated design guidelines and take-away messages, Fig. 33 portrays the factors affecting the inevitable trade-offs. More explicitly, we can always improve the $\mathrm{BE}$ by compromising the $\mathrm{PE}$, since a higher-throughput modulation scheme requires higher power. One can also improve the BER and Symbol Error Ratio (SER) at the cost of higher TX power, but this would improve the eavesdropper's chances of intercepting confidential information. The BER/SER can also be improved by using more complex FEC codes and receivers as well as Automatic Repeat Request (ARQ) schemes, but all these sophisticated techniques tend to increase the processing complexity and hence also the Digital-Signal-Processing (DSP) scheme's power dissipation.

The outer shell of Fig. 33 portrays a suite of the LACOOFDM enabling techniques and parameters, which determine the overall system performance. To elaborate on their effect on the trade-offs seen in the central core of Fig. 33, we list a few of the most influential design factors:

1) Again, increasing the number of bits/symbol can be readily achieved by using high-throughput QAM schemes, which require increased TX power and more complex DSP. This improves the BE.

2) The throughput can also be increased by using more layers in conjugation with more complex DSP and higher power dissipation, i.e. lower PE.

3) The BER/SER can be substantially improved by FEC coding at the cost of reducing the effective throughput, owing to the redundant parity bits. Both block codes and convolutional style codes (CC) can be used, but the family of CC-style codes are more amenable to powerful iterative detection, as detailed in earlier in Section V. Both the specific choice of the code (CC, TC, BoseChaudhuriHocquenghem $[\mathrm{BCH}]$ codes, ReedSolomon $[\mathrm{RS}]$ codes, polar codes, Low-Density Parity-Check [LDPC] codes, etc.) as well as their code-rate and decoding algorithms directly affect the BER vs. throughput vs. delay vs. complexity relationship. Similar trends are valid for the choice of the channel interleaver and the ARQ schemes used, just to mention a few of the intricate design trade-offs.

\section{Future Research Challenges}

We provide a selection of potential future research directions below.

Downlink/Uplink Decoupling: Although both downlink and uplink VLC transmissions have been investigated in [90]-[92] and substantial efforts have also been dedicated to bidirectional VLC transmissions [93], [94], much of the research has been focused on the downlink. This is partially due to the challenges of uplink VLC implementations. Hence, RF WiFi solutions have been adopted as the uplink counterpart of a VLC downlink [95], which decouples the transmission links and creates Heterogeneous Networks (HetNets). This also allows the entire WiFi-band to be used for the uplink. Apart from its simpler implementation and lower deployment costs [96], RF uplink solutions have been shown to have lower transmit power requirements and/or better SNR [97], hence resulting in higher data rates [98]. Therefore, further research should be devoted to the design of a HetNet RF/VLC system relying on LACO-OFDM, where the remaining cooperation and scheduling challenges have to be tackled.

Power Line Backhaul: Apart from the uplink issues discussed above, the backhaul also plays an important role in VLC networks, which should ideally be provided by Power Line Communications (PLC) [99] instead of laying an extra Ethernet link. However, typical PLC transmissions are susceptible both to the impulse noise and to multipath reflections, which are commonly found in this hostile medium [100], [101], creating a unique type of system, which requires dedicated tools for equalizing the channel effects and mitigating the errors. As a result, despite the huge throughput potential of VLC, the PLC-based backhaul requires much more research attention. Therefore, a joint system design must be conceived, considering both the end user requirements and the PLC backhaul. Fortunately, the flexibility of LACO-OFDM provides us with increased design freedom, thanks to its flexible BE vs. PE trade-off. Another option is to power the LED lights via the Ethernet cable, which is a much more friendly transmission medium than the PLC channel [102].

Fiber Optic Communications: To expound a little further, a more reliable but more costly wired communications technique of connecting the LED transmitters is to harness optical fibers, which have a higher throughput than PLC. Experiments have been conducted using LACO-OFDM signals over short-haul $(\sim 20 \mathrm{~km})$ fiber-optic links having data rates ranging from $4.375 \mathrm{~Gb} / \mathrm{s}$ [46], [103] up to $9.375 \mathrm{~Gb} / \mathrm{s}$ [86]. These experiments have demonstrated notable improvements in bandwidth efficiency over conventional optic-fiber links based mainly on DCO-OFDM. However, similarly to PLC, optical-fiber channels also have their unique impairments, hence requiring further research.

Multiuser LACO-OFDM: Originally conceived way back in the 1960s [104], Multiple-Input Multiple-Output (MIMO) systems have been widely studied in the RF field [105][108] for supporting simultaneous communications for multiple users within the same spectral resources. More recently, 
adopting MultiUser MIMO (MU-MIMO) techniques for VLC systems has also attracted much attention [109], [110] for further improving the system's performance. However, due to the unique structure of LACO-OFDM and its requirement of layer-by-layer detection at the receiver, conventional OMA techniques cannot be readily applied, since each user requires the knowledge of all users' information for canceling the clipping distortion in each layer. As a remedy, the NOMA concept may be harnessed [111] for supporting multiple users with the aid of LACO-OFDM, where other users' information can either be canceled out or regarded as noise [56]. However, scheduling and power allocation still remain challenging issues, when applying NOMA techniques in MU-MIMO LACOOFDM systems, which requires further investigations.

Outdoor VLC: At the time of writing, the vast majority of research on VLC has been dedicated to indoor scenarios, given the vulnerability of optical signals to the shot noise induced by the filtered sunlight [112]. However, given the recent development of sophisticated techniques and the better understanding of the outdoor channel characteristics [113], powerful adaptive bandwidth based counter-measurements have been proposed for improving the performance and for mitigating the shot noise [114]. More recently, the performance of the basic ACO-OFDM and DCO-OFDM schemes operating in outdoor scenarios have also been characterized [115], calling for more sophisticated, robust and power-efficient schemes relying on strong FEC techniques, such as our multi-class coded LACOOFDM scheme of Sec. V.

\section{APPENDIX A}

\section{Proof of THE LOSSLESS CLIPPING CHARACTERISTIC OF LACO-OFDM}

In this section, we provide the mathematical proof of the fact that in LACO-OFDM, the clipping distortion of each layer falls only on the null subcarriers controlled by the same layer. The $k$ th subcarrier is controlled by layer $l$, if and only if we have:

$$
\bmod \left(k, 2^{l-1}\right)=0 .
$$

It is worth mentioning that LACO-OFDM inherited these characteristics from ACO-OFDM. Therefore, these conclusions also apply to ACO-OFDM by letting $l=1$.

Theorem 1 (Lossless Clipping in LACO-OFDM). The clipping distortion of layer $l$ in LACO-OFDM only falls onto the subcarriers indexed by

$$
k=u \cdot 2^{l}, 0 \leq u \leq \frac{K}{2^{l}}-1 .
$$

Proof. The theorem mentioned above states the fact that no clipping distortion can be found on the rest of the subcarriers controlled by layer $l$, where

$$
k^{\prime}=u \cdot 2^{l}+2^{l-1}, 0 \leq u \leq \frac{K}{2^{l}}-1 .
$$

The corresponding symbols on these subcarriers after clipping can be expressed as

$$
\mathcal{F}\left\{\boldsymbol{s}_{l}\right\}\left[k^{\prime}\right]=\frac{1}{\sqrt{K}} \sum_{n=0}^{K-1} s_{l}[n] W_{K}^{-k^{\prime} n} .
$$

If we segment the summation of (20) into $\frac{K}{K_{l}}=2^{l-1}$ parts, each of which adds up $K_{l}$ subcarriers, then we have:

$$
\begin{aligned}
& \mathcal{F}\left\{\boldsymbol{s}_{l}\right\}\left[k^{\prime}\right] \\
= & \frac{1}{\sqrt{K}}\left(\sum_{n=0}^{K_{l}-1} s_{l}[n] W_{K}^{-k^{\prime} n}+\sum_{n=K_{l}}^{2 K_{l}-1} s_{l}[n] W_{K}^{-k^{\prime} n}+\ldots\right. \\
& \left.+\sum_{n=b K_{l}}^{(b+1) K_{l}-1} s_{l}[n] W_{K}^{-k^{\prime} n}+\cdots+\sum_{n=K-K_{l}}^{K-1} s_{l}[n] W_{K}^{-k^{\prime} n}\right) .
\end{aligned}
$$

Replacing $n$ by $\left(n+b K_{l}\right)$ in the $(b+1)$ st summation yields

$$
\begin{aligned}
& \mathcal{F}\left\{\boldsymbol{s}_{l}\right\}\left[k^{\prime}\right] \\
& =\sum_{b=0}^{2^{l-1}-1}\left[\frac{1}{\sqrt{K}} \sum_{n=0}^{K_{l}-1}\left(s_{l}\left[n+b K_{l}\right] W_{K}^{-k^{\prime}\left(n+b K_{l}\right)}\right)\right] \\
& \stackrel{(24)}{=} \frac{1}{\sqrt{K}} \sum_{n=0}^{K_{l}-1}\left[s_{l}[n] W_{K}^{-k^{\prime} n}\left(\sum_{b=0}^{2^{l-1}-1} W_{K}^{\left.-b k^{\prime} K_{l}\right)}\right)\right] \\
& \stackrel{(23)}{=} \frac{B_{l, k^{\prime}}}{\sqrt{K}} \sum_{n=0}^{K_{l}-1} s_{l}[n] W_{K}^{-k^{\prime} n},
\end{aligned}
$$

where

$$
\begin{aligned}
B_{l, k^{\prime}} & =\sum_{b=0}^{2^{l-1}-1} W_{K}^{-b k^{\prime} K_{l}}=\sum_{b=0}^{2^{l-1}-1} \exp \left[\frac{-b k^{\prime} K_{l}}{K}\right] \\
& =\sum_{b=0}^{2^{l-1}-1} \exp \left[\frac{-b k^{\prime}}{2^{l-1}}\right]
\end{aligned}
$$

is a constant only dependent on $l$ and $k^{\prime}$, thus can be taken outside. The second equality in (22) takes advantage of the repetition in the layer signal formulated as:

$$
s_{l}[n]=s_{l}\left[n+K_{l}\right]=s_{l}\left[n+2 K_{l}\right]=\ldots
$$

The final summation in (22) can be further split into two parts as in (25), where

$$
\begin{aligned}
W_{K}^{-\frac{k^{\prime} K_{l}}{2}} & =\exp \left[-j \frac{2 \pi}{K} \frac{k^{\prime} K_{l}}{2}\right] \\
& \stackrel{(19)}{=} \exp \left[-j \frac{2 \pi}{K} \frac{\left(2^{l} u+2^{l-1}\right)\left(K / 2^{l-1}\right)}{2}\right] \\
& =\exp [-j \pi(2 u+1)] \equiv-1, \forall u \in \mathbb{Z} .
\end{aligned}
$$

Bearing in mind that the this equality only holds when $(2 u+1)$ is an odd number, namely that this only happens to fall on subcarriers loaded with the current layer's symbols or their conjugates, we have:

$$
\mathcal{F}\left\{\boldsymbol{s}_{l}\right\}\left[k^{\prime}\right]=\frac{B_{l, k^{\prime}}}{\sqrt{K}} \sum_{n=0}^{\frac{K_{l}}{2}-1}\left(s_{l}[n]-s_{l}\left[\frac{K_{l}}{2}+n\right]\right) W_{K}^{-k^{\prime} n} .
$$

Given the asymmetry of ACO-OFDM signal specified before, for each element in the summation of (27), either $s_{l}[n]$ or $s_{l}\left[\frac{K_{l}}{2}+n\right]$ should be zero.

- If we have $s_{l}\left[\frac{K_{l}}{2}+n\right]=0$, then $\bar{s}_{l}\left[\frac{K_{l}}{2}+n\right]$ in the unclipped signal $\bar{s}_{l}$ must be negative and must have been 


$$
\begin{aligned}
\mathcal{F}\left\{\boldsymbol{s}_{l}\right\}\left[k^{\prime}\right] & =\frac{B_{l, k^{\prime}}}{\sqrt{K}}\left(\sum_{n=0}^{\frac{K_{l}}{2}-1} s_{l}[n] W_{K}^{-k^{\prime} n}+\sum_{n=\frac{K_{l}}{2}}^{K_{l}-1} s_{l}[n] W_{K}^{-k^{\prime} n}\right) \\
& =\frac{B_{l, k^{\prime}}}{\sqrt{K}}\left(\sum_{n=0}^{\frac{K_{l}}{2}-1} s_{l}[n] W_{K}^{-k^{\prime} n}+\sum_{n=0}^{\frac{K_{l}}{2}-1} s_{l}\left[\frac{K_{l}}{2}+n\right] W_{K}^{\left.-k^{\prime}\left(\frac{K_{l}}{2}+n\right)\right)}\right. \\
& =\frac{B_{l, k^{\prime}}}{\sqrt{K}}\left(\sum_{n=0}^{\frac{K_{l}}{2}-1} s_{l}[n] W_{K}^{-k^{\prime} n}+\sum_{n=0}^{\frac{K_{l}}{2}-1} s_{l}\left[\frac{K_{l}}{2}+n\right] W_{K}^{-k^{\prime} n} W_{K}^{-\frac{k^{\prime} K_{l}}{2}}\right)
\end{aligned}
$$

clipped. Therefore, based on (2), $\bar{s}_{l}[n]$ should be positive and retained after clipping, yielding:

$$
s[n]=\bar{s}_{l}[n]=-\bar{s}_{l}\left[\frac{K_{l}}{2}+n\right]=\bar{s}_{l}\left[\frac{K_{l}}{2}+n\right] W_{K}^{-\frac{k^{\prime} K_{l}}{2}} .
$$

Substituting this back to (27) gives (29) shown on the next page, where the equality on the 2 nd line of (29) replaces the two $s_{l}[n] \mathrm{s}$ by $\bar{s}_{l}$ and $\bar{s}_{l}\left[\frac{K_{l}}{2}+n\right] W_{K}^{-\frac{k^{\prime} K_{l}}{2}}$, respectively, according to (28), while the equality on the 4th line of (29) substitutes $n$ in the second summation by $\left(n-\frac{K_{l}}{2}\right)$. Substituting $B_{l, k^{\prime}}$ back into the summation can expand the summation range back up to $(K-1)$ :

$$
\begin{aligned}
& \mathcal{F}\left\{\boldsymbol{s}_{l}\right\}\left[k^{\prime}\right] \\
& =\frac{1}{2} \frac{1}{\sqrt{K_{l}}} \sum_{n=0}^{\frac{K_{l}}{2}-1} \bar{s}_{l}[n] W_{K}^{-k^{\prime} n} B_{l, k^{\prime}} \\
& =\frac{1}{2} \frac{1}{\sqrt{K_{l}}} \sum_{n=0}^{\frac{K_{l}}{2}-1}\left(\bar{s}_{l}[n] W_{K}^{-k^{\prime} n} \sum_{b=0}^{2^{l-1}-1} W_{K}^{-b k^{\prime} K_{l}}\right) \\
& =\frac{1}{2} \frac{1}{\sqrt{K_{l}}} \sum_{b=0}^{2^{l-1}-1} \sum_{n=0}^{\frac{K_{l}}{2}-1} \bar{s}_{l}\left[n+b K_{l}\right] W_{K}^{-k^{\prime}\left(n+b K_{l}\right)} \\
& =\frac{1}{2} \frac{1}{\sqrt{K}} \sum_{n=0}^{K-1} \bar{s}_{l}[n] W_{K}^{-k^{\prime} n}=\frac{1}{2} \overline{\boldsymbol{S}}_{l}\left[k^{\prime}\right] .
\end{aligned}
$$

- If $s_{l}[n]=0$, we can arrive at

$$
\begin{aligned}
& -s_{l}\left[\frac{K_{l}}{2}+n\right]=-\bar{s}_{l}\left[\frac{K_{l}}{2}+n\right] \\
& =\bar{s}_{l}\left[\frac{K_{l}}{2}+n\right] W_{K}^{-\frac{k^{\prime} K_{l}}{2}}=\bar{s}_{l}[n] .
\end{aligned}
$$

Substituting this back into (27) leads to

$$
\begin{aligned}
& \mathcal{F}\left\{s_{l}\right\}\left[k^{\prime}\right]=\frac{B_{l, k^{\prime}}}{\sqrt{K}} \sum_{n=0}^{\frac{K_{l}}{2}-1}\left(-s_{l}\left[\frac{K_{l}}{2}+n\right]\right) W_{K}^{-k^{\prime} n} \\
& =\frac{B_{l, k^{\prime}}}{2 \sqrt{K}} \sum_{n=0}^{\frac{K_{l}}{2}-1}\left(\bar{s}_{l}[n]+\bar{s}_{l}\left[\frac{K_{l}}{2}+n\right] W_{K}^{-\frac{k^{\prime} K_{l}}{2}}\right) W_{K}^{-k^{\prime} n},
\end{aligned}
$$

which takes the track of the maths back to that of (29) in the previous case, because the result is the same as
TABLE $X$

MAXIMUM BANDWIDTH EFFICIENCY OF O-OFDM SCHEMES

\begin{tabular}{lr}
\hline Scheme & $\mathrm{BE}_{\max }(\mathrm{bps} / \mathrm{Hz})$ \\
\hline ACO-OFDM & 1.5 \\
2-Layer LACO-OFDM & 2.25 \\
3-Layer LACO-OFDM & 2.625 \\
4-Layer LACO-OFDM & 2.8125 \\
5-Layer LACO-OFDM & 2.90625 \\
DCO-OFDM & 3 \\
\hline
\end{tabular}

the second line of (29). Naturally, the same conclusion as (30) can be given.

It has now been shown that only the subcarriers with indices $k^{\prime}$ satisfying (19) have their energy halved after the clipping process, with no information loss observed. Therefore, the clipping distortion can only fall onto other subcarriers controlled by the layer, which follows the rule of (18).

\section{APPENDIX B}

\section{DEFINITION OF THE POWER AND BANDWIDTH EFFICIENCY}

In Fig. 5, we highlighted the trade-offs between the BE, PE and BER of different O-OFDM signals. The schemes shown in Fig. 5 include DCO-OFDM, ACO-OFDM and LACO-OFDM having 2, 3, 4 and 5 layers. It is worth mentioning that ACOOFDM can be considered as a special case of 1-layer LACOOFDM. All schemes considered in this figure use 64QAM, while the DCO-OFDM scheme has a DC offset of $13 \mathrm{~dB}$.

In this paper, we define the BE of a signal as the actual number of bit conveyed per channel use at a certain BER. In an error-free transmission, the maximum $\mathrm{BE}\left(\mathrm{BE}_{\max }\right)$ can be attained, which is only related to the bit-to-symbol mapping scheme (64QAM in this case) used and the composition of the signal, which is listed below in Tab. X. The instantaneous $\mathrm{BE}$ at a certain BER would be calculated based on $\mathrm{BE}_{\max }$ as

$$
\mathrm{BE}=\mathrm{BE}_{\max } \cdot \mathrm{BER} .
$$

On the other hand, we define PE as the amount of power (Watt) used for conveying a single bit of information per channel use. Therefore, the standard unit of PE is given by bit/Watt, while Fig. 5 uses bits per nanoWatt (bit/nW). For DCO-OFDM, this also includes the power assigned to the DC offset. Explicitly, the PE is equal to

$$
\mathrm{PE}=\mathrm{BE} / E_{s}
$$




$$
\begin{aligned}
& \mathcal{F}\left\{\boldsymbol{s}_{l}\right\}\left[k^{\prime}\right]=\frac{B_{l, k^{\prime}}}{\sqrt{K}} \sum_{n=0}^{\frac{K_{l}}{2}-1} s_{l}[n] W_{K}^{-k^{\prime} n}=\frac{B_{l, k^{\prime}}}{2 \sqrt{K}} \sum_{n=0}^{\frac{K_{l}}{2}-1}\left(s_{l}[n]+s_{l}[n]\right) W_{K}^{-k^{\prime} n} \\
& =\frac{B_{l, k^{\prime}}}{2 \sqrt{K}} \sum_{n=0}^{\frac{K_{l}}{2}-1}\left(\bar{s}_{l}[n]+\bar{s}_{l}\left[\frac{K_{l}}{2}+n\right] W_{K}^{-\frac{k^{\prime} K_{l}}{2}}\right) W_{K}^{-k^{\prime} n} \\
& =\frac{B_{l, k^{\prime}}}{2 \sqrt{K}}\left(\sum_{n=0}^{\frac{K_{l}}{2}-1} \bar{s}_{l}[n] W_{K}^{-k^{\prime} n}+\sum_{n=0}^{\frac{K_{l}}{2}-1} \bar{s}_{l}\left[\frac{K_{l}}{2}+n\right] W_{K}^{-k^{\prime}\left(\frac{K_{l}}{2}+n\right)}\right) \\
& =\frac{B_{l, k^{\prime}}}{2 \sqrt{K}}\left(\sum_{n=0}^{\frac{K_{l}}{2}-1} \bar{s}_{l}[n] W_{K}^{-k^{\prime} n}+\sum_{n=\frac{K_{l}}{2}}^{K_{l}-1} \bar{s}_{l}[n] W_{K}^{-k^{\prime} n}\right) \\
& =\frac{B_{l, k^{\prime}}}{2 \sqrt{K}} \sum_{n=0}^{\frac{K_{l}}{2}-1} \bar{s}_{l}[n] W_{K}^{-k^{\prime} n}
\end{aligned}
$$

where $\mathrm{BE}$ is given in (33), and $E_{s}$ is the average electric signal power in Watt corresponding to the BER used for obtaining the $\mathrm{BE}$, when the AWGN power is $-10 \mathrm{dBm}$.

\section{REFERENCES}

[1] R. W. Chang, "Orthogonal frequency multiplex data transmission system," Jan. 1970. US Patent 3,488,445.

[2] J. Salz and S. Weinstein, "Fourier transform communication system," in Proc. 1st ACM Symp. Problems Optim. Data Commun. Syst., (Pine Mountain, GA, USA), pp. 99-128, ACM, Oct. 1969.

[3] A. Peled and A. Ruiz, "Frequency domain data transmission using reduced computational complexity algorithms," in Proc. '80 IEEE Int. Conf. Acoust. Speech Signal Process., vol. 5, (Denver, CO, USA), pp. 964-967, 1980.

[4] L. Cimini, "Analysis and simulation of a digital mobile channel using orthogonal frequency division multiplexing," IEEE Trans. Commun., vol. 33, pp. 665-675, Jul. 1985.

[5] R. Lassalle and M. Alard, "Principles of modulation and channel coding for digital broadcasting for mobile receivers," EBU Tech. Rev, vol. 224, no. 1, pp. 68-190, 1987.

[6] L. Hanzo, M. Münster, B. Choi, and T. Keller, OFDM and MC-CDMA for broadband multi-user communications, WLANs and broadcasting. Chichester, U.K.: Wiley, 2005.

[7] L. Hanzo, Y. Akhtman, J. Akhtman, L. Wang, and M. Jiang, MIMOOFDM for LTE, WiFi and WiMAX: Coherent versus non-coherent and cooperative turbo transceivers. Chichester, U.K.: Wiley, 2011.

[8] J. Armstrong, "OFDM for optical communications," J. Lightw. Technol., vol. 27, pp. 189-204, Feb. 2009.

[9] S. Feng, X. Li, R. Zhang, M. Jiang, and L. Hanzo, "Hybrid positioning aided amorphous-cell assisted user-centric visible light downlink techniques," IEEE Access, vol. 4, pp. 2705-2713, 2016.

[10] Z. Wang, Q. Wang, W. Huang, and Z. Xu, Visible light communications: Modulation and signal processing. Hoboken, NJ, USA: Wiley, 2018.

[11] S. Feng, R. Zhang, X. Li, Q. Wang, and L. Hanzo, "Dynamic throughput maximization for the user-centric visible light downlink in the face of practical considerations," IEEE Trans. Wireless Commun., vol. 17, pp. 5001-5015, Aug. 2018

[12] J. M. Kahn and J. R. Barry, "Wireless infrared communications," Proc. IEEE, vol. 85, pp. 265-198, Feb. 1997.

[13] L. Hanzo, H. Haas, S. Imre, D. O'Brien, M. Rupp, and L. Gyongyosi, "Wireless myths, realities, and futures: From $3 \mathrm{G} / 4 \mathrm{G}$ to optical and quantum wireless," Proc. IEEE, vol. 100, pp. 1853-1888, May 2012.

[14] P. H. Pathak, X. Feng, P. Hu, and P. Mohapatra, "Visible light communication, networking, and sensing: A survey, potential and challenges," IEEE Commun. Surv. Tuts., vol. 17, pp. 2047-2077, 4th Quart. 2015.

[15] J. Zhou, Y. Yan, Z. Cai, Y. Qiao, and Y. Ji, "A cost-effective and efficient scheme for optical OFDM in short-range IM/DD systems," IEEE Photon. Technol. Lett., vol. 26, pp. 1372-1374, Jul. 2014.
[16] J. B. Carruthers and J. M. Kahn, "Multiple-subcarrier modulation for nondirected wireless infrared communication," IEEE J. Sel. Areas Commun., vol. 14, pp. 538-546, Apr. 1996.

[17] O. González, R. Perez-Jimenez, S. Rodriguez, J. Rabadán, and A. Ayala, "OFDM over indoor wireless optical channel," IEE Proc. Optoelectronics, vol. 152, pp. 199-204, Aug. 2005.

[18] J. Armstrong and A. J. Lowery, "Power efficient optical OFDM," Electron. Lett., vol. 42, Mar. 2006.

[19] J. Armstrong and B. J. C. Schmidt, "Comparison of asymmetrically clipped optical OFDM and DC-biased optical OFDM in AWGN," IEEE Commun. Lett., vol. 12, pp. 343-345, May 2008.

[20] S. Lee, S. Randel, F. Breyer, and A. Koonen, "PAM-DMT for intensitymodulated and direct-detection optical communication systems," IEEE Photon. Technol. Lett., vol. 21, pp. 1749-1751, Dec. 2009.

[21] N. Fernando, Y. Hong, and E. Viterbo, "Flip-OFDM for unipolar communication systems," IEEE Trans. Commun., vol. 60, pp. 37263733, Dec. 2012.

[22] D. Tsonev, S. Sinanovic, and H. Haas, "Novel unipolar orthogona frequency division multiplexing (U-OFDM) for optical wireless," in Proc. IEEE 75th Veh. Technol. Conf. (VTC Spring), (Yokohama, Japan), pp. 1-5, May 2012.

[23] R. Bai, Q. Wang, and Z. Wang, "Asymmetrically clipped absolute value optical OFDM for intensity-modulated direct-detection systems," J. Lightw. Technol., vol. 35, pp. 3680-3691, Sep. 2017.

[24] S. D. Dissanayake, K. Panta, and J. Armstrong, "A novel technique to simultaneously transmit ACO-OFDM and DCO-OFDM in IM/DD systems," in Proc. 2011 IEEE Glob. Commun. Conf. Workshop, (Houston, TX, USA), pp. 782-786, Dec. 2011.

[25] B. Ranjha and M. Kavehrad, "Hybrid asymmetrically clipped OFDMbased IM/DD optical wireless system," J. Opt. Commun. Netw., vol. 6 , pp. 387-396, Apr. 2014.

[26] N. Wu and Y. Bar-Ness, "A novel power-efficient scheme asymmetrically and symmetrically clipping optical (ASCO)-OFDM for IM/DD optical systems," EURASIP J. Adv. Signal Process., vol. 2015, pp. 110, Jan. 2015.

[27] Q. Wang, Z. Wang, and L. Dai, "Asymmetrical hybrid optical ofdm for visible light communications with dimming control," IEEE Photon. Technol. Lett., vol. 27, pp. 974-977, May 2015.

[28] S. D. Dissanayake and J. Armstrong, "Comparison of ACO-OFDM, DCO-OFDM and ADO-OFDM in IM/DD systems," J. Lightw. Technol., vol. 31, pp. 1063-1072, Apr. 2013.

[29] Q. Wang, C. Qian, X. Guo, Z. Wang, D. G. Cunningham, and I. H. White, "Layered ACO-OFDM for intensity-modulated direct-detection optical wireless transmission," Opt. Express, vol. 23, pp. 12382-12393, May 2015.

[30] I. A. Hemadeh, M. El-Hajjar, and L. Hanzo, "Hierarchical multifunctional layered spatial modulation," IEEE Access, vol. 6, pp. 94929533, 2018.

[31] S. Gopi, S. Kalyani, and L. Hanzo, "Coherent and non-coherent multilayer index modulation," IEEE Access, vol. 7, pp. 79677-79693, 2019. 
[32] R. Zhang and L. Hanzo, "Multi-layer modulation for intensitymodulated direct-detection optical OFDM," J. Opt. Commun. Netw., vol. 5, pp. 1402-1412, Dec. 2013.

[33] D. Tsonev and H. Haas, "Avoiding spectral efficiency loss in unipolar OFDM for optical wireless communication," in Proc. 2014 IEEE Int. Conf. Commun. (ICC), (Sydney, NSW, Australia), pp. 3336-3341, Jun. 2014

[34] D. Tsonev, S. Videv, and H. Haas, "Unlocking spectral efficiency in intensity modulation and direct detection systems," IEEE J. Sel. Areas Commun., vol. 33, pp. 1758-1770, Sep. 2015.

[35] H. Elgala and T. D. C. Little, "SEE-OFDM: Spectral and energy efficient OFDM for optical IM/DD systems," in Proc. 2014 IEEE 25th Annu. Int. Symp. Pers. Indoor Mobile Radio Commun. (PIMRC), (Washington, DC, USA), pp. 851-855, Sep. 2014.

[36] M. S. Islim, D. Tsonev, and H. Haas, "On the superposition modulation for OFDM-based optical wireless communication," in Proc. 2015 IEEE Global Conf. Signal Inf. Process. (GlobalSIP), (Orlando, FL, USA), pp. 1022-1026, Dec. 2015.

[37] M. S. Islim, D. Tsonev, and H. Haas, "Spectrally enhanced PAM-DMT for IM/DD optical wireless communications," in Proc. 2015 IEEE 26th Annu. Int. Symp. Pers. Indoor Mobile Radio Commun. (PIMRC), (Hong Kong, China), pp. 877-882, 30 Aug.-2 Sep. 2015.

[38] A. J. Lowery, "Enhanced asymmetrically clipped optical ODFM for high spectral efficiency and sensitivity," in Proc. 2016 Opt. Fiber Commun. Conf. Exhib. (OFC), (Anaheim, CA, USA), pp. 1-3, Mar. 2016

[39] M. Z. Afgani, H. Haas, H. Elgala, and D. Knipp, "Visible light communication using OFDM," in Proc. 2nd Int. Conf. Testbeds Res. Infrastructures Develop. Netw. Communities (TRIDENTCOM), (Barcelona, Spain), p. 6, Mar. 2006.

[40] J. Grubor, V. Jungnickel, and K.-D. Langer, "Adaptive optical wireless OFDM system with controlled asymmetric clipping," in Conf. Rec. 41st Asilomar Conf. Signals Syst. Comput., (Pacific Grove, CA, USA), pp. 1896-1902, Nov. 2007.

[41] Q. Wang, Z. Wang, X. Guo, and L. Dai, "Improved receiver design for layered ACO-OFDM in optical wireless communications," IEEE Photon. Technol. Lett., vol. 28, pp. 319-322, Feb. 2016.

[42] T. Q. Wang, H. Li, and X. Huang, "Diversity combining for layered asymmetrically clipped optical OFDM using soft successive interference cancellation," IEEE Commun. Lett., vol. 21, pp. 1309-1312, Jun. 2017.

[43] M. A. Mohammed, C. He, and J. Armstrong, "Diversity combining in layered asymmetrically clipped optical OFDM," J. Lightw. Technol., vol. 35, pp. 2078-2085, Jun. 2017.

[44] Y. Sun, F. Yang, and J. Gao, "Comparison of hybrid optical modulation schemes for visible light communication," IEEE Photon. J., vol. 9, pp. 1-13, Jun. 2017.

[45] X. Zhang, Q. Wang, R. Zhang, S. Chen, and L. Hanzo, "Performance analysis of layered ACO-OFDM," IEEE Access, vol. 5, pp. 18366$18381,2017$.

[46] B. Song, B. Corcoran, Q. Wang, L. Zhuang, and A. J. Lowery, "Subcarrier pairwise coding for short-haul L/E-ACO-OFDM," IEEE Photon. Technol. Lett., vol. 29, pp. 1584-1587, Sep. 2017.

[47] F. Yang, Y. Sun, and J. Gao, "Adaptive LACO-OFDM with variable layer for visible light communication," IEEE Photon. J., vol. 9, pp. 1-8, Dec. 2017.

[48] J. Zhou, Q. Wang, Q. Cheng, M. Guo, Y. Lu, A. Yang, and Y. Qiao, "Low-PAPR layered/enhanced ACO-SCFDM for optical-wireless communications," IEEE Photon. Technol. Lett., vol. 30, pp. 165-168, Jan. 2018.

[49] J. Zhou and W. Zhang, "A comparative study of unipolar OFDM schemes in Gaussian optical intensity channel," IEEE Trans. Commun., vol. 66, pp. 1549-1564, Apr. 2018.

[50] T. Q. Wang, H. Li, and X. Huang, "Interference cancellation for layered asymmetrically clipped optical OFDM with application to optical receiver design," J. Lightw. Technol., vol. 36, pp. 2100-2113, Jun. 2018.

[51] Y. Sun, F. Yang, and L. Cheng, "An overview of OFDM-based visible light communication systems from the perspective of energy efficiency versus spectral efficiency," IEEE Access, vol. 6, pp. 60824-60833, 2018.

[52] T. Q. Wang, H. Li, and X. Huang, "Analysis and mitigation of clipping noise in layered ACO-OFDM based visible light communication systems," IEEE Trans. Commun., vol. 67, pp. 564-577, Jan. 2019.

[53] X. Zhang, Z. Babar, S. Chen, and L. Hanzo, "Multi-class coded layered asymmetrically clipped optical OFDM," IEEE Trans. Commun., vol. 67 , pp. 578-589, Jan. 2019.
[54] T. Zhang, Y. Qiao, J. Zhou, Z. Zhang, Y. Lu, and F. Su, "Spectralefficient L/E-ACO-SCFDM-based dimmable visible light communication system," IEEE Access, vol. 7, pp. 10617-10626, 2019.

[55] B. Li, W. Xu, S. Feng, and Z. Li, "Spectral-efficient reconstructed LACO-OFDM transmission for dimming compatible visible light communications," IEEE Photonics J., vol. 11, pp. 1-14, Feb. 2019.

[56] S. Feng, R. Zhang, W. Xu, and L. Hanzo, "Multiple access design for ultra-dense VLC networks: orthogonal vs non-orthogonal," IEEE Trans. Commun., vol. 67, pp. 2218-2232, Mar. 2019.

[57] H. Li, Z. Huang, Y. Xiao, S. Zhan, and Y. Ji, "A power and spectrum efficient NOMA scheme for VLC network based on hierarchical predistorted LACO-OFDM," IEEE Access, vol. 7, pp. 48565-48571, 2019.

[58] Z. Babar, X. Zhang, P. Botsinis, D. Alanis, D. Chandra, S. X. Ng, and L. Hanzo, "Near-capacity multilayered code design for LACO-OFDMaided optical wireless systems," IEEE Trans. Veh. Technol., vol. 68, pp. 4051-4054, Apr. 2019.

[59] R. Bai and S. Hranilovic, "Absolute value layered ACO-OFDM for intensity-modulated optical wireless channels," in 2019 IEEE Int. Conf. Commun. (ICC), (Shanghai, China), pp. 1-6, May 2019.

[60] A. W. Azim, Y. Le Guennec, and G. Maury, "Performance analysis of precoded layered ACO-OFDM for visible light communication systems," Opt. Commun., vol. 440, pp. 49-60, Jun. 2019.

[61] H. Ji, S. Qiao, and T. Zhang, "A MISO-VLC system based on LACOOFDM and superposed constellation demodulation," in 2019 9th Int. Conf. Info. Sci. Technol. (ICIST), (Hulunbuir, China), pp. 294-298, Aug. 2019

[62] Y. Sun and F. Yang, "Adaptive modulation scheme based on partially pre-distorted LACO-OFDM for VLC system," IEEE Photonics J., vol. 11, pp. 1-12, Oct. 2019.

[63] T. Zhang, H. Ji, Z. Ghassemlooy, X. Tang, B. Lin, and S. Qiao, "Spectrum-efficient triple-layer hybrid optical OFDM for IM/DD-based optical wireless communications," IEEE Access, vol. 8, pp. 1035210362,2020

[64] W. Hu, "Design of a cyclic shifted LACO-OFDM for optical wireless communication," IEEE Access, vol. 8, pp. 76708-76714, 2020.

[65] H. Ji, B. Lin, X. Tang, and J. Xu, "Triple-layer hybrid optical OFDM scheme for passive optical network," Opt. Commun., p. 125622, Jul. 2020.

[66] M. Abd Elkarim, M. Elsherbini, H. M. AbdelKader, and M. H. Aly, "Exploring the effect of LED nonlinearity on the performance of layered ACO-OFDM," Appl. Opt., vol. 59, pp. 7343-7351, Aug. 2020.

[67] X. Liu, J. Li, J. Li, and Z. Huang, "Analysis of the single-fft receiver for layered aco-ofdm in visible light communications," Journal of Lightwave Technology, vol. 38, pp. 4757-4764, Sep. 2020.

[68] X. Zhang, S. Chen, and L. Hanzo, "On the discrete-input continuousoutput memoryless channel capacity of layered ACO-OFDM," $J$. Lightw. Technol., vol. 38, pp. 4955-4968, Sep. 2020.

[69] C. Lacava, Z. Babar, X. Zhang, I. Demirtzioglou, P. Petropoulos, and L. Hanzo, "High-speed multi-layer coded adaptive laco-ofdm and its experimental verification," OSA Continuиm, vol. 3, pp. 2614-2629, Sep. 2020

[70] Z. Zhang, A. Chaaban, and M.-S. Alouini, "Residual clipping noise in multi-layer optical OFDM: Modeling, analysis, and applications," IEEE Trans. Wireless Commun., vol. 19, pp. 5846-5859, Sep. 2020.

[71] X. Yan, J. He, J. Shi, Z. Zhou, and Q. Tang, "Time-efficient adaptive modulation scheme for LACO-OFDM in VLC systems," Opt. Commun., p. 126069, Nov. 2020.

[72] A. W. Azim, Y. Le Guennec, M. Chafii, and L. Ros, "LACO-OFDM with index modulation for optical wireless systems," IEEE Wireles. Commun. Lett., 2020. early access.

[73] S. Vappangi and V. Mani, "Concurrent illumination and communication: A survey on visible light communication," Physical Commun., vol. 33, pp. 90-114, Apr. 2019.

[74] A. J. Lowery, "Spectrally efficient optical orthogonal frequency division multiplexing," Philos. Trans. Roy. Soc. A, vol. 378, p. 20190180, Mar. 2020.

[75] S. Tian, K. Panta, H. A. Suraweera, B. J. S. J. Schmidt, S. McLaughlin, and J. Armstrong, "A novel timing synchronization method for ACOOFDM-based optical wireless communications," IEEE Trans. Wireless Commun., vol. 7, pp. 4958-4967, Dec. 2008.

[76] T. Keller and L. Hanzo, "Adaptive multicarrier modulation: A convenient framework for time-frequency processing in wireless communications," Proc. IEEE, vol. 88, pp. 611-640, May 2000.

[77] T. Keller, L. Piazzo, P. Mandarini, and L. Hanzo, "Orthogonal frequency division multiplex synchronization techniques for frequencyselective fading channels," IEEE J. Sel. Areas Commun., vol. 19, pp. 999-1008, Jun. 2001. 
[78] R. Mesleh, H. Elgala, and H. Haas, "Performance analysis of indoor OFDM optical wireless communication systems," in Proc. 2012 IEEE Wireless Commun. Netw. Conf., (Paris, France), pp. 1005-1010, Apr. 2012.

[79] A. Goldsmith, Wireless Communications. Cambridge, U.K.: Cambridge Univ. Press, 1st ed., 2005

[80] L. Hanzo, F. C. A. Somerville, and J. Woodard, Voice and audio compression for wireless communications. Chichester, U.K.: Wiley, 2nd ed., 2008.

[81] J. Jiang, Y. Huo, F. Jin, P. Zhang, Z. Wang, Z. Xu, H. Haas, and L. Hanzo, "Video streaming in the multiuser indoor visible light downlink," IEEE Access, vol. 3, pp. 2959-2986, 2015.

[82] Y. Zhang, I. A. Hemadeh, M. El-Hajjar, and L. Hanzo, "Multi-set space-time shift keying assisted adaptive inter-layer FEC for wireless video streaming," IEEE Access, vol. 7, pp. 3592-3609, 2018.

[83] H. Chen, R. G. Maunder, and L. Hanzo, "An EXIT-chart aided design procedure for near-capacity N-component parallel concatenated codes," in 2010 IEEE Global Commun. Conf. (GLOBECOM), (Miami, FL, USA), pp. 1-5, Dec. 2010.

[84] H. Chen, R. G. Maunder, and L. Hanzo, "Low-complexity multiplecomponent turbo-decoding-aided hybrid ARQ," IEEE Trans. Veh. Technol., vol. 60, pp. 1571-1577, May 2011.

[85] S. ten Brink, "Convergence of iterative decoding," Electron. Lett., vol. 35, pp. 806-808, May 1999.

[86] Q. Wang, B. Song, B. Corcoran, D. Boland, C. Zhu, L. Zhuang, and A. J. Lowery, "Hardware-efficient signal generation of layered/enhanced ACO-OFDM for short-haul fiber-optic links," Opt. Express, vol. 25, pp. 13359-13371, Jun. 2017.

[87] L. Dai, B. Wang, Y. Yuan, S. Han, I. Chih-Lin, and Z. Wang, "Nonorthogonal multiple access for 5G: Solutions, challenges, opportunities, and future research trends," IEEE Commun. Mag., vol. 53, pp. 74-81, Sep. 2015.

[88] Y. Saito, Y. Kishiyama, A. Benjebbour, T. Nakamura, A. Li, and K. Higuchi, "Non-orthogonal multiple access (NOMA) for cellular future radio access," in 2013 IEEE 77th vehicular technology conference (VTC Spring), (Dresden, Germany), pp. 1-5, IEEE, Jan. 2013.

[89] L. Hao, D. Wang, W. Cheng, J. Li, and A. Ma, "Performance enhancement of ACO-OFDM-based VLC systems using a hybrid autoencoder scheme," Opt. Commun., vol. 442, pp. 110-116, Jul. 2019.

[90] B. Lin, X. Tang, H. Yang, Z. Ghassemlooy, S. Zhang, Y. Li, and C. Lin, "Experimental demonstration of IFDMA for uplink visible light communication," IEEE Photon. Technol. Lett., vol. 28, pp. 2218-2220, Oct. 2016.

[91] Y. F. Liu, C. H. Yeh, C. W. Chow, Y. Liu, Y. L. Liu, and H. K. Tsang, "Demonstration of bi-directional LED visible light communication using TDD traffic with mitigation of reflection interference," Opt. Express, vol. 20, pp. 23019-23024, Oct. 2012.

[92] Y. Yang, Z. Zeng, J. Cheng, C. Guo, and C. Feng, "A relay-assisted OFDM system for VLC uplink transmission," IEEE Trans. Commun., vol. 67, pp. 6268-6281, Sep. 2019.

[93] M. D. Soltani, X. Wu, M. Safari, and H. Haas, "Bidirectional user throughput maximization based on feedback reduction in LiFi networks," IEEE Trans. Commun., vol. 66, pp. 3172-3186, Jul. 2018.

[94] M. D. Soltani, M. A. Arfaoui, I. Tavakkolnia, A. Ghrayeb, M. Safari, C. M. Assi, M. O. Hasna, and H. Haas, "Bidirectional Optical Spatial Modulation for Mobile Users: Toward a Practical Design for LiFi Systems," IEEE J. Sel. Areas Commun., vol. 37, pp. 2069-2086, Sep. 2019.

[95] L. Feng, R. Q. Hu, J. Wang, P. Xu, and Y. Qian, "Applying VLC in 5G networks: Architectures and key technologies," IEEE Netw., vol. 30, pp. 77-83, Nov./Dec. 2016.

[96] F. Boccardi, J. Andrews, H. Elshaer, M. Dohler, S. Parkvall, P. Popovski, and S. Singh, "Why to decouple the uplink and downlink in cellular networks and how to do it," IEEE Commun. Mag., vol. 54 pp. 110-117, Mar. 2016

[97] K. Smiljkovikj, P. Popovski, and L. Gavrilovska, "Analysis of the decoupled access for downlink and uplink in wireless heterogeneous networks," IEEE Wireless Commun. Lett., vol. 4, pp. 173-176, Jan. 2015

[98] S. Singh, X. Zhang, and J. G. Andrews, "Joint rate and SINR coverage analysis for decoupled uplink-downlink biased cell associations in HetNets," IEEE Trans. Wireless Commun., vol. 14, pp. 5360-5373, Oct. 2015.

[99] S. Feng, T. Bai, and L. Hanzo, "Joint Power Allocation for the MultiUser NOMA-Downlink in a Power-Line-Fed VLC Network," IEEE Trans. Veh. Technol., vol. 68, pp. 5185-5190, May 2019.
[100] N. Taherinejad, L. Lampe, and S. Mirabbasi, "An adaptive impedancematching system for vehicular power line communication," IEEE Trans. Veh. Technol., vol. 66, pp. 927-940, Feb. 2017.

[101] M. Zimmermann and K. Dostert, "A multipath model for the powerline channel," IEEE Trans. Commun., vol. 50, pp. 553-559, Apr. 2002.

[102] F. Delgado, I. Quintana, J. Rufo, J. Rabadan, C. Quintana, and R. PerezJimenez, "Design and implementation of an Ethernet-VLC interface for broadcast transmissions," IEEE Commun. Lett., vol. 14, pp. 1089-1091, Dec. 2010.

[103] B. Song, C. Zhu, B. Corcoran, Q. Wang, L. Zhuang, and A. J. Lowery, "Experimental layered/enhanced ACO-OFDM short-haul optical fiber link," IEEE Photon. Technol. Lett., vol. 28, pp. 2815-2818, Dec. 2016.

[104] D. Shnidman, "A generalized nyquist criterion and an optimum linear receiver for a pulse modulation system," Bell Syst. Tech. J., vol. 46, pp. 2163-277, Nov. 1967

[105] S. Yang and L. Hanzo, "Fifty years of MIMO detection: The road to large-scale MIMOs," IEEE Commun. Surv. Tuts., vol. 17, pp. 19411988, 4th Quart. 2015.

[106] S. Lu, I. A. Hemadeh, M. El-Hajjar, and L. Hanzo, "Compressed sensing-aided multi-dimensional index modulation," IEEE Trans. Commun., vol. 67, pp. 4074-4087, Jun. 2019.

[107] S. Lu, I. A. Hemadeh, M. El-Hajjar, and L. Hanzo, "An adaptive multi-user mimo scheme for the millimeter-wave downlink," in Proc 2018 IEEE 29th Annu. Int. Symp. Pers. Indoor Mobile Radio Commun. (PIMRC), (Bologna, Italy), pp. 1-5, 2018.

[108] H. Liu, S. Lu, M. El-Hajjar, and L.-L. Yang, "Machine learning assisted adaptive index modulation for mmWave communications," IEEE Open J. Commun. Soc., vol. 1, pp. 1425-1441, 2020.

[109] Q. Wang, Z. Wang, and L. Dai, "Multiuser MIMO-OFDM for visible light communications," IEEE Photon. J., vol. 7, pp. 1-11, Jun. 2015.

[110] F. Wang, C. Liu, Q. Wang, J. Zhang, R. Zhang, L.-L. Yang, and L. Hanzo, "Optical jamming enhances the secrecy performance of the generalized space-shift-keying-aided visible-light downlink," IEEE Trans. Commun., vol. 66, pp. 4087-4102, Sep. 2018.

[111] Y. Liu, Z. Qin, M. Elkashlan, Z. Ding, A. Nallanathan, and L. Hanzo, "Nonorthogonal multiple access for $5 \mathrm{G}$ and beyond," Proc. IEEE, vol. 105, pp. 2347-2381, Dec. 2017.

[112] A.-M. Căilean and M. Dimian, "Current challenges for visible light communications usage in vehicle applications: A survey," IEEE Commun. Surv. Tuts., vol. 19, pp. 2681-2703, 3rd Quart. 2017.

[113] M. S. Islim, S. Videv, M. Safari, E. Xie, J. J. McKendry, E. Gu, M. D. Dawson, and H. Haas, "The impact of solar irradiance on visible light communications," J. Lightw. Technol., vol. 36, pp. 2376-2386, Jun. 2018.

[114] Y. H. Chung and S.-b. Oh, "Efficient optical filtering for outdoor visible light communications in the presence of sunlight or articifical light," in Proc. 2013 Int. Symp. Intell. Signal Process. Commun. Syst. (ISPACS), (Naha, Japan), pp. 749-752, Nov. 2013

[115] B. G. Guzmán, V. P. G. Jiménez, M. C. Aguayo-Torres, H. Haas, and L. Hanzo, "Downlink performance of optical ofdm in outdoor visible light communication," IEEE Access, vol. 6, pp. 76854-76866, 2018

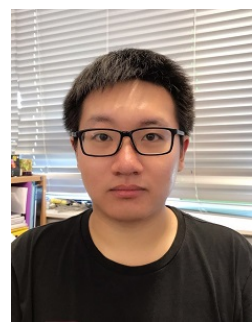

Xiaoyu Zhang (Member, IEEE) received the B.Eng. degree in electronic information engineering from the University of Electronic Science and Technology of China (UESTC), Chengdu, China, in 2014, and the M.Sc. and Ph.D. degrees from the University of Southampton, Southampton, U.K., in 2015 and 2020, respectively. He is currently a Research Fellow in Optical Wireless Communications at the University of Southampton, where he is affiliated with both the Next Generation Wireless Group and the Optoelectronics Research Centre. His research interests include the modulation, signal processing, performance analysis and optimization for wireless and optical communications. 


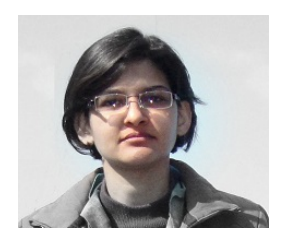

Zunaira Babar (Senior Member, IEEE) received her B.Eng. degree in electrical engineering from the National University of Science \& Technology (NUST), Islamabad, Pakistan, in 2008, and the M.Sc. degree (Distinction) and the Ph.D degree in wireless communications from the University of Southampton, U.K., in 2011 and 2015, respectively. She is currently working as an Algorithms Design Engineer at Viavi solutions and is an Adjunct Research Fellow in the Next Generation Wireless group at the University of Southampton.

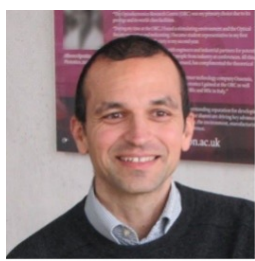

Periklis Petropoulos graduated from the Department of Electrical Engineering and Information Technology, University of Patras. He received an MSc degree in Communications Engineering from the University of Manchester Institute of Science and Technology and a PhD degree in Optical Telecommunications from the Optoelectronics Research Centre (ORC), University of Southampton, where he currently works as a Professor. His research interests lie in the fields of optical communications, all-optical signal processing and novel fibre and waveguide technologies and he leads national research projects in these areas. His research has been published extensively in technical journals and conference proceedings, including several invited and post-deadline papers at major international conferences.

Prof Petropoulos is the Editor-in-Chief of the IET Optoelectronics technical journal. He has served both as member and sub-committee chair of the Technical Programme Committees for several international conferences, including the European Conference on Optical Communication (ECOC) and the Optical Fiber Communication (OFC) conference. He is a Fellow of the Optical Society of America.

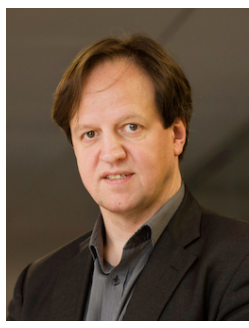

Harald Haas FREng FRSE FIEEE FIET received the $\mathrm{PhD}$ degree from The University of Edinburgh in 2001. He is a Distinguished Professor of Mobile Communications at the University of Strathclyde and the Director of the LiFi Research and Development Centre. He also set-up and co-founded pureLiFi Ltd which it currently serves as Chief Scientific Officer $\mathrm{He}$ has authored over 550 conference and journal papers. Haas main research interests are in optical wireless communications, hybrid optical wireless and RF communications, spatial modulation, and interference coordination in wireless networks. His team invented spatial modulation. He introduced LiFi to the public at an invited TED Global talk in 2011. LiFi was listed among the 50 best inventions in TIME Magazine in 2011. He gave a second TED Global lecture in 2015 on the use of solar cells as LiFi data detectors and energy harvesters. In 2016, he received the Outstanding Achievement Award from the International Solid State Lighting Alliance. In 2019 he was recipient of IEEE Vehicular Society James Evans Avant Garde Award. Haas was elected a Fellow of the Royal Society of Edinburgh (RSE) in 2017. In the same year he received a Royal Society Wolfson Research Merit Award and was elevated to IEEE Fellow. In 2018 he received a three-year EPSRC Established Career Fellowship extension and was elected Fellow of the IET. Haas was elected Fellow of the Royal Academy of Engineering in 2019.

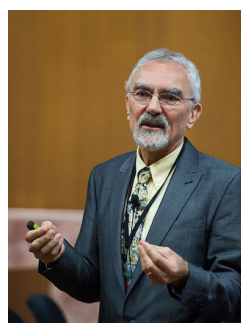

Lajos Hanzo (Fellow,

IEEE)

(http://www-mobile.ecs.soton.ac.uk/,

https://en.wikipedia.org/wiki/Lajos_Hanzo) received his Master degree and Doctorate in 1976 and 1983, respectively from the Technical University (TU) of Budapest. He was also awarded the Doctor of Sciences (DSc) degree by the University of Southampton (2004) and Honorary Doctorates by the TU of Budapest (2009) and by the University of Edinburgh (2015). He is a Foreign Member of the Hungarian Academy of Sciences and a former Editor-in-Chief of the IEEE Press. He has served several terms as Governor of both IEEE ComSoc and of VTS. He has published 1900+ contributions at IEEE Xplore, 19 Wiley-IEEE Press books and has helped the fast-track career of $123 \mathrm{PhD}$ students. Over 40 of them are Professors at various stages of their careers in academia and many of them are leading scientists in the wireless industry. He is also a Fellow of the Royal Academy of Engineering (FREng), of the IET and of EURASIP. 